\title{
Santilli's Isodual Mathematics and Physics for Antimatter
}

\author{
P. M. Bhujbal \\ Department of Physics, Nutan Adarsh Arts, Commerce and Smt. Maniben Harilal Wegad Science College, Umrer, India
}

Email address:

prashantmbhujbal@yahoo.com

\section{To cite this article:}

P. M. Bhujbal. Santilli's Isodual Mathematics and Physics for Antimatter. American Journal of Modern Physics. Special Issue: Issue II: Foundations of Hadronic Mechanics. Vol. 5, No. 2-1, 2016, pp. 161-184. doi: 10.11648/j.ajmp.2016050201.20

Received: July 3, 2015; Accepted: July 4, 2015; Published: June 1, 2016

\begin{abstract}
In this paper, we study the isodual branch of hadronic mechanics achieved by the Italian-American scientist R. M. Santilli following decades of research. We show that, thanks to the background isodual mathematics (whose knowledge is assumed), Santilli's isodual theory of antimatter appears to be the only axiomatically consistent and time invariant theory permitting a \{lit classical\} representation of \{ \it neutral\} (as well as charged) antimatter whose operator image is equivalent to charge conjugation, thus verifying all known experimental data in the field. We then show the important prediction of \{lit gravitational repulsion $\}$ between matter and antimatter, with particular reference to the prediction that light emitted by antimatter, known as \{ \it isodual light, \} experiences gravitational repulsion when in the field of matter. We finally point out that recent detections of antimatter galaxies via Santilli telescope with concave lenses may eventually result to be the first experimental evidence of antigravity.
\end{abstract}

Keywords: Antimatter, Isodual Light, Antigravity

\section{Introduction}

After being conjectured by A. Schuster in 1898, antimatter was predicted by P. A. M. Dirac [1] in the late 1920's in the negative-energy solutions of his celebrated equation. Dirac himself soon discovered that particles with negative-energy do not behave in a physical way and, for this reason, he submitted his celebrated "hole theory," which subsequently restricted the study of antimatter to the sole level of second quantization [2]. This occurrence created an imbalance in the physics of this century because matter is described at all levels of study, from Newtonian mechanics to quantum field theory, while antimatter is solely treated at the level of second quantization.

To initiate the study for the future removal of this imbalance in due time, Santilli presented a theory of antimatter which has been conceived to begin at the purely classical Newtonian level, and then to admit corresponding images at all subsequent levels of study [3] in which the guiding principle is to identify a map which possesses the main mathematical structure of charge conjugation, yet it is applicable at all levels, and not solely at the operator level.

The main characteristic of charge conjugation is that of being antiautomorphic (where the term "automorphic" is referred to the map of a given space onto itself). After studying a number of possibilities, Santilli has selected a map which is anti-isomorphic (where the term "isomorphic" is referred to a map from one space onto another of equivalent topological characteristics to be identified later on) applicable at all levels of study, and given by the following isodual map here generically expressed to an arbitrary quantity Q (i.e. a function, or a matrix or an operator)

$$
\mathrm{Q}(\mathrm{x}, \varphi, \ldots) \rightarrow \mathrm{Q}^{\mathrm{d}}\left(\mathrm{x}^{\mathrm{d}}, \varphi^{\mathrm{d}}, \ldots\right)=-\mathrm{Q}^{\dagger}\left(-\mathrm{x}^{\dagger},-\varphi^{\dagger}, \ldots\right)
$$

which, for consistency, must be applied to the totality of the mathematical structure of the conventional theory of matter, including numbers, fields, spaces, geometries, algebras, etc. This results in a new mathematics, called isodual mathematics, which is at the foundation of the classical isodual theory of antimatter of this paper.

The isodual map was first proposed by Santilli [4] in 1985 and then remained ignored for several years. According to it the isoduality can be represented as $+1 \rightarrow 1^{\mathrm{d}}=-1$. The first hypothesis on the isodual theory of antimatter appeared for the operator version in 1993 [5] which also contains an initial study of the equivalence between isoduality and charge conjugation. The prediction of the isodual theory that antimatter in the field of matter experiences antigravity was first submitted in 1994 [6] which also proposed an experiment 
for the measure of the gravity of elementary antiparticles in the gravitational field of earth. The experiment essentially consists of comparative measurements under the gravity of collimated, low energy beams of positrons and electrons in horizontal flight on a tube with sufficiently high vacuum as well as protection from stray and patch fields and of sufficient length to permit a definite result, e.g. the view by the naked eye of the displacements due to gravity of the positron and electron beams on a scintillator at the end of the flight.

Theoretical and experimental studies on the isodual theory of antimatter were conducted at the International Workshop on Antimatter Gravity and Anti-Hydrogen Atom Spectroscopy, held in Sepino, Italy, in May 1996 [7].

The motivations for the classical isodual theory of antimatter are rather numerous. First, there is the need indicated earlier to achieve a full equivalence in the treatment of matter and antimatter beginning at the classical level. In fact, far away galaxies and quasars may well be made up of antimatter. The absence of a classical theory of antimatter therefore implies the evident impossibility of quantitative studies of this important astrophysical issue.

Second, the current gravitational treatment of antimatter is afflicted by a number of problematic aspects. Current theories are based on only one map from classical to operator settings, the naive or symplectic quantization. Therefore, conventional classical representations of antimatter via positive energies do not yield antiparticles under quantization, but conventional particles with the mere reversal of the sign of the charge.

Third, there is a fundamental incompatibility between current theories of gravitation and unified gauge theories of electroweak interactions which is due precisely to antimatter. In fact, current gravitational theories characterize antimatter via a positive-definite energy-momentum tensor, while electroweak theories characterize antiparticles via negative energy states. Additional motivations have been identified in [8-10]. The need for a systematic study aiming at a resolution of these issues is then beyond scientific doubts.

The isodual theory emerged from the identification of negative units in the antiparticle component of the conventional Dirac equation and the reconstruction of the theory with respect to that unit. Isoduality therefore provides a mere reinterpretation of Dirac's original notion of antiparticle, while leaving all numerical predictions under electroweak interactions essentially unchanged.

Santilli was able to construct the isodual theory of antimatter which characterizes antimatter at all possible levels, from Newtonian mechanics to second quantization on the basis of his isodual mathematics [11-13].

In particular, isodual mathematics permitted the first known geometrically consistent representation of the gravitational field of a neutral antimatter body via the Riemann-Santilli isodual geometry defined over isodual fields $[11,13]$.

It should be stressed that the isodual theory of antimatter requires, for consistency requirement, the conjugation of all physical quantities of matter as well as, most importantly, all their units of measurements. Consequently, antimatter evolves along a time moving backward, the $\mathrm{t}^{\mathrm{d}}=-\mathrm{t}$, and has negative-definite energy $\mathrm{E}^{\mathrm{d}}=-\mathrm{E}$ along Dirac's original conception [14]. The historical inconsistencies are resolved via the joint conjunction of the related units; in fact, negative time and negative energy referred to negative units of time and energy are as causal as our positive time and energies referred to positive units of time and energy.

Eventually, in this paper the author tried to focus on some important features of Santilli's Isodual Physics for antimatter based on Santilli's Isodual Mathematics.

\section{Salient Features of Santilli's Isodual Mathematics}

The Newton's equations, Galileo's relativity and Einstein's special and general relativities were conceived before the discovery of antimatter and therefore no classical representation of "neutral" antimatter could be generated since at that time only conjugation from matter to antimatter was the change of sign of the charge [15], which created one of the biggest scientific imbalance in the history because throughout the $20^{\text {th }}$ century matter was studied at all possible levels, from Newtonian mechanics to second quantization, while antimatter was solely studied at the particle level. In essence, the prevalent stand still adopted is that, since Einstein's special and general relativities do not provide a proper description, antimatter does not exist in the universe in any appreciable amount. The sole generally admitted exception is that of man-made antiparticles created in laboratory, since their existence cannot be denied.

The above scientific imbalance was identified, apparently for the first time, by the Italian-American scientist Ruggero Maria Santilli. Santilli has been interested since his graduate studies to ascertain whether a far away galaxy is made up of matter or of antimatter. He soon learned that Newtonian, Galilean and Einsteinian theories had no value for the indicated problem since far away galaxies must be assumed to be neutral, in which case said theories had no distinction whatsoever between matter and antimatter. For this reason, Santilli initiated a long journey that first required the identification of mathematical means for the consistent classical distinction between neutral matter and antimatter prior to any possible physical application. Santilli discovered that a mathematics for the consistent classical treatment of neutral (or charged) antimatter did not exist and had to be built.

The $20^{\text {th }}$ century position on antimatter implied the rather general belief that antimatter galaxies do not exist. This stringent stand eliminated altogether the problem of detecting antimatter asteroids on grounds that they do not exist due to the absence of the antimatter galaxies and related antimatter supernovas needed for their origination. This position was evidently based in the unspoken intent of maintaining the validity of Einstein's theories for all of the universe via the denial of the existence of antimatter galaxies, despite it being disproved by evidence since our Earth has indeed been hit in the past by devastating antimatter asteroids, and similar 
asteroids have been detected by various observatories.

In fact, the catastrophic 1908 Tunguska explosion in Siberia with the power of one thousand Hiroshima nuclear bombs can be solely interpreted in a scientific way as being due to an antimatter asteroid annihilating in our atmosphere $[15,16]$. This is due to various reasons, such as the complete absence of debris, let alone of a crater, in the ground. The Tunguska explosion excited the entire Earth's atmosphere for days, to such an extent those two days following the explosion; people could read newspapers in Sydney, Australia, at midnight without artificial light; and other reasons. Such a large excitation of the atomic and molecular constituents of our atmosphere can only be scientifically (i.e. quantitatively) represented as being due to huge radiations that, in turn, can only originate from the annihilation of an antimatter asteroid with our matter atmosphere. The widely accepted "interpretation" of the Tunguska explosion as being due to a (matter) comet has no scientific credibility due to the impossibility of such an origination to excite the entire Earth's atmosphere for days, and occur with the absence of debris in the grounds, let alone with the absence of a crater.

NASA has also reported explosions in our upper atmosphere that can only be due to small antimatter asteroids because annihilating at the time of contact with the upper portion of our matter atmosphere. Similarly, astronauts and cosmonauts have observed ashes in our upper atmosphere when on the dark side with respect to our Sun; these ashes can be best interpreted as being due to antimatter cosmic rays that annihilate in our atmosphere, because the only cosmic rays that can reach us at sea level being those due to matter cosmic rays.

As indicated above as well as earlier by Santilli and others, the existence of antimatter stars and galaxies is imperative and should not be ignored. As a representative example out of many, that recall antimatter is thought to exist in the Oort cloud in view of a possible explanation for gamma ray bursts. In fact, these phenomena can be explained by the annihilation of matter and antimatter asteroids or small comets. The explosion would create powerful gamma ray bursts and accelerate matter [17].

Besides antimatter asteroids, it is possible that Earth has been hit in the past by antimatter comets as indicated by the old observations, since the biblical times, not only of excessive brilliance but also of trajectories in our atmosphere that cannot be interpreted as being due to matter comets, e.g., because of slow penetration of the said objects in our atmosphere. In conclusion, the evidence on the existence of antimatter asteroids as well as of antimatter comets and their possibility of hitting Earth again is sufficiently serious [18].

Scientific studies in the detection of antimatter asteroids requires mathematical and physical theories suitable for the classical treatment of neutral antimatter evidently because antimatter asteroids are too large to be treated via operator theories and they must be assumed as being neutral since they are isolated in space. Santilli has repeatedly stated in his writings that: A protracted lack of solution of physical problems is generally due to the use of insufficient or inadequate mathematics [15]. Additionally, Santilli stated that: There cannot exist a really new physical theory without a really new mathematics, and there cannot exist a really new mathematics without new numbers [loc. cit.]. For this reason, Santilli had spent decades in purely mathematical research, firstly, to identify new numbers and, secondly, to develop new mathematics that would allow a classical treatment of neutral or charged antimatter, because the entire body of applied mathematics is built on numbers.

Along these lines, the most fundamental and very first paper published by Santilli on antimatter is Ref. [19] of 1993 that introduced for the first time new numbers called "isodual" where the prefix "iso" was introduced in the Greek sense of indicating the preservation of conventional axioms used for matter and the term "dual" stands to indicate the map from matter to antimatter. The role of Santilli isodual numbers is such that his entire theory of antimatter that is called "isodual" precisely because of the main character of the new basic numbers.

It should be noted that Santilli discovered the new isodual number in Ref. [19] as a particular case of much more general numbers he called "isonumbers" and "genonumbers" and their isoduals.

Following the discovery of new numbers, Santilli constructed in Ref. [20] also of 1993 the isodualities of the Euclidean and Minkowski spaces which were evidently needed for any possible physical applications. He then proceeded in Ref. [21] to the construction of the isodual image of Lie's theory because it is evidently necessary for the construction of basic symmetries for antimatter, viz. the isodual images of the rotation, Galileo and Lorentz symmetries.

Finally, in the mathematical memoir [22] Santilli made a second fundamental mathematical discovery, a new formulation of the ordinary differential calculus that resulted in being crucial for the achievement of the first known formulation of Newton's equations for neutral or charged antiparticles. The complete formulation of the novel isodual mathematics was first presented by Santilli in monograph [8] of 1994 and then updated in monographs [15] of 2001.

Following the achievement of structural consistency of the new isodual mathematics, and only thereafter, Santilli initiated his physical studies with paper [23] of 1993 written on his original aim of the 1960s as a graduate student, how to detect possible antimatter stars and galaxies.

It is evident that the problem of detecting possible antimatter asteroids is of such a magnitude that it cannot be left unaddressed just to maintain the validity of Einstein's theories for antimatter. In an event North America is hit by an antimatter asteroid even with the size of a football, all North American communications will be disrupted, while the Military will be inoperative for days, due to extreme radiations absorbed and re-emitted by Earth's atmosphere. The same holds in the unfortunate event an antimatter asteroids hits India, Russia, China or other regions. Consequently, the problem of possible antimatter asteroids requires attention not only by the people at large, but also by the scientific and 
military communities.

The multitude of open problems created by the detection of antimatter have been studied for decades by Santilli who has provided scientific arguments establishing that the threat to Earth caused by antimatter asteroids is more serious than what popularly believed in contemporary academia.

Therefore, Santilli had to confront the problem of identifying a mathematical conjugation (also called map or duality) capable of performing the transition from matter to antimatter at the purely classical Newtonian level, irrespectively of whether matter and antimatter are neutral or charged, under the condition that such a map recovers charge conjugation at the quantum level for the sake of consistency, which is evidently needed.

Following a decade of unpublished trials and errors, Santilli selected the following main assumption for the construction of the needed new mathematics. Recall that the conventional charge conjugation is defined on a Hilbert space $\mathrm{H}$ with states $\psi(\mathrm{r})$ over the field of complex numbers $\mathrm{C}$ and can be characterized by a conjugation of the type applied to the quantum representation of matter

$$
\mathrm{C} \psi(\mathrm{r})=-\psi^{\dagger}(\mathrm{r})
$$

where $\mathrm{r}$ is the coordinate of the Euclidean representation space.

Consequently, Santilli introduced an anti-Hermitean conjugation called isoduality and denoted with the upper index $d$ that, by central condition, has to be applied to all physical quantities, to all their units and to all their operations, and can be written as,

$$
\mathrm{Q}(\mathrm{t}, \mathrm{r}, \mathrm{v}, \ldots) \rightarrow \mathrm{Q}^{\mathrm{d}}\left(\mathrm{t}^{\mathrm{d}}, \mathrm{r}^{\mathrm{d}}, \mathrm{v}^{\mathrm{d}} \ldots\right)=-\mathrm{Q}^{\dagger}\left(-\mathrm{t}^{\dagger},-\mathrm{r}^{\dagger},-\mathrm{v}^{\dagger, \ldots}\right)
$$

where $\mathrm{Q}$ denotes a generic quantity depending on time $\mathrm{t}$, coordinates $\mathrm{r}$, velocities $\mathrm{v}$, and any other needed variables [15].

For the trivial case of real numbers, isoduality reduces to the mere change of the sign of all quantities, all their units and the related operations. In the event a given body is charged, the isoduality evidently also applies by changing the sign of the charge. Hence, Santilli's isoduality applies irrespective of whether the body is charged or not.

The main difference between conventional charge conjugation and Santilli's isoduality is that the former solely applies at the quantum level, while the latter applies, by central conception, at the classical Newtonian level, as well as at all subsequent levels of study, including the quantum level in which charge conjugation and isoduality are equivalent [15].

In terms as simple as permitted by the advanced nature of the topic, the classical isodual map from neutral matter to the corresponding neutral antimatter requires that all physical quantities change their sign. Consequentially, under the isodual map, time, energy, linear momentum, entropy, and other positive-definite physical quantities, become negative. There have been a number of proposals that antimatter must move backward in time as a condition to admit annihilation into light. This rather natural assumption has been dismissed due to the violation of causality when motion backward in time is treated with the mathematics used for matter [15].

However, for Santilli's isodual mathematics, antimatter's motion backward in time $t^{\mathrm{d}}=-\mathrm{t}<0$, when referred to a negative unit of time (e.g., $\mathrm{s}^{\mathrm{d}}=-1 \mathrm{~s}$ ), is as causal as matter moving forward in our time $t>0$ when referred to the usual positive units of time (e.g., +1 s) [19].

Similarly, it is known since Dirac's time that negative energies also violate causality laws. However, Santilli has shown that negative-definite isodual energies $\mathrm{E}^{\mathrm{d}}=-\mathrm{E}<0$ referred to negative units (e.g., $\mathrm{erg}^{\mathrm{d}}=-1 \mathrm{erg}$ ) are as physical as conventional positive energies $\mathrm{E}>0$ referred to positive units (e.g., $+1 \mathrm{erg}$ ). Inconsistencies emerge only under crossovers of the two worlds, such as when positive energies are measured with negative units and vice versa [19].

In going deeper into the problem, Santilli discovered that the correct formulation of antimatter requires an entire new mathematics, today known as Santilli's isodual mathematics, which can be defined as the anti-Hermitean image of the entire mathematics used for matter, thus including isodual numbers, isodual functional analysis, isodual differential calculus, isodual Lie's theory, etc. In fact, any mix-up, even minute, of the mathematics for matter and that for antimatter leads to catastrophic inconsistencies that are generally not realized by non-experts in the field [19].

Santilli's research in antimatter was delayed for years by the classification of all "numbers" (namely, sets verifying the axioms of a numeric field) into real, complex and quaternion numbers that had been achieved by historic masters such as Gauss, Cayley, Hamilton and others. Finally, in 1993 Santilli re-inspected this historical classification and discovered that the axioms of a numeric field do not require the basic multiplicative unit to be necessarily positive definite, since said unit can be negative definite as well, provided that the conventional associative product of numbers $\mathrm{n} \times \mathrm{m}$ is redefined in such a way to admit the newly assumed negative unit [19].

We have in this way Santilli's isodual fields $F^{\mathrm{d}}\left(\mathrm{n}^{\mathrm{d}}, \mathrm{x}^{\mathrm{d}}, 1^{\mathrm{d}}\right)$ consisting of isodual real, isodual complex and isodual quaternion numbers with negative-definite isodual multiplicative unit and related isodual (associative) multiplication [19]

$F^{d}\left(n^{d}, x^{d}, 1^{d}\right): n^{d}=-n^{\dagger}, n^{d} x^{d} m^{d}=n^{d} x\left(1^{d}\right)^{-1} \times m^{d}, 1^{d}=-1^{\dagger},(4)$

under which $1^{\mathrm{d}}$ is indeed the basic multiplicative unit at all levels (the additive unit 0 of a field remains evidently unchanged under isoduality because $0^{\mathrm{d}} \equiv 0$ [19]),

$$
1^{\mathrm{d}} \mathrm{x}^{\mathrm{d}} \mathrm{n}^{\mathrm{d}} \equiv \mathrm{n}^{\mathrm{d}} \mathrm{x}^{\mathrm{d}} 1^{\mathrm{d}} \forall \mathrm{n}^{\mathrm{d}} \varepsilon \mathrm{F}^{\mathrm{d}},
$$

Note, to prevent insidious misinterpretations, that is for the evident mathematical consistency, all real numerical values in isodual mathematics must be elements of Santilli's isodual fields and, as such, must be given by ordinary numbers multiplied by the isodual unit.

Consequently, Santilli undertook to the construction of 
isodual functional analysis and isodual metric spaces, such as

the Euclid-Santilli isodual space with line element [20]

$$
E^{d}\left(r^{d}, \delta^{d}, I^{d}\right): r^{d}=r \times I^{d}, \delta^{d}=\text { Diag. }(-1,-1,-1)=\delta \times I^{d}, I^{d}=\text { Diag. }(-1,-1,-1),
$$

$$
r^{\mathrm{d} 2 \mathrm{~d}}=\left(\mathrm{r}^{\mathrm{di}} \mathrm{x}^{\mathrm{d}} \delta_{\mathrm{ij}}^{\mathrm{d}} \mathrm{x}^{\mathrm{d}} \mathrm{r}^{\mathrm{dj}}\right) \times \mathrm{I}^{\mathrm{d}} \equiv \mathrm{r}^{2}=\left(\mathrm{r}^{\mathrm{i}} \times \delta_{\mathrm{ij}} \times \mathrm{r}^{\mathrm{j}}\right) \times \mathrm{I}^{\mathrm{d}},
$$

where one should note the necessity of multiplying the line element by the isodual unit as a condition to be an isodual scalar, that is, an element of the isodual field. It then follows that the line element of the Euclid-Santilli isodual space coincides with that of the conventional Euclidean space, Eq. (7), and this explains the reason for the lack of detection of the isodual spaces for centuries [20].

Note that the study of the isodualities of the Euclidean space was a necessary pre-requisite to reach the yet unknown (in 1993) formulation of Newton's equation for neutral or charged antimatter [20].

Additionally, Santilli constructed the isoduality of Lie's theories, Minkowskian and Riemannian geometries and of virtually all mathematics used for the study of matter [21].

Thanks to his keen self-criticism, the emerging new mathematics continued to have hidden inconsistencies whose solution required additional years of study. Finally, in 1995, Santilli had the courage to re-inspect another pillar of $20^{\text {th }}$ century applied mathematics, the ordinary differential calculus, by discovering that, contrary to popular belief since Newton's time, the differential calculus does indeed depend on the assumed basic unit and related field. We reach in this way the discovery of Santilli's isodual differential calculus with isodual differentials and isodual derivatives that, for the case of a real-valued new unit acquires the simple form [22]

$$
\begin{aligned}
& d^{d} r^{d}=1^{d} d r^{d}=1^{d} d\left(r 1^{d}\right) \equiv d r \\
& \delta^{d} F^{d}\left(r^{d}\right) /{ }^{d} \delta^{d} r^{d}=-\delta F / \delta r
\end{aligned}
$$

where one can see that (again for the case of a real-valued new unit) Santilli's isodual differential coincides with the conventional differential, and this explains the reason the isodual differential calculus remained unidentified for centuries.

Following the discovery of the isodual differential calculus, Santilli completed the construction of the isodual mathematics with a rigorous structural consistency, and passed only thereafter to physical applications.

A fundamental prediction of the isodual theory of antimatter is that light emitted by antimatter (antimatter light) is physically different than light emitted by matter (matter light) in a number of experimentally verifiable ways [9]. This important prediction was presented by Santilli at the International Conference on Antimatter held in Sepino, Italy, on June 1996.

Recall that light has no charge. But the isodual theory has been constructed to provide a differentiation between neutral matter and antimatter. Therefore, the physical distinction between matter and antimatter light is a direct and unavoidable consequence of the physical distinction between neutral matter and antimatter, as reflected in the distinction between their representations.

Besides, the physical differentiations at advanced level we cannot review here, the most visible difference is the prediction by the isodual theory of antimatter that antimatter light is repelled by a matter gravitational field (see Figure 9).

The simplest way to illustrate this prediction is that at the primitive Newtonian level since all subsequent levels of study are merely consequential. Let us recall that Santilli has formulated Newtonian gravitation in a truly "universal" way via the "identical" representation of the historical equation in terms of "energy," rather than mass [8, 24].

Following the study of a number of alternatives, Santilli gave priority to the search for new numbers since all mathematics used for physics must be based on a numeric field as a condition for experimental verifications and, in any case, all aspects of applied mathematics can be built on a given numeric field via simple compatibility arguments. In 1993, Santilli $[19,25]$ finally identified the desired new number under the name of isodual real, complex and quaternionic numbers [17], which verify the condition of being anti-isomorphic to the conventional real, complex and quaternionic numbers, respectively. The word "isodual" was suggested to indicate a duality under the preservation of the conventional abstract axioms of numeric fields. The crucial condition of anti-isomorphism was achieved via the anti-Hermitean conjugation of all elements of a numeric field and all its operations. This implies that, given a field $\mathrm{F}(\mathrm{n}, \times, 1)$ with elements $n, m, \ldots$, conventional associative product $n \times m$ $=\mathrm{nm}$ and trivial unit 1 , Santilli isodual fields are indicated with the upper symbols $\mathrm{d}, \mathrm{F}^{\mathrm{d}}\left(\mathrm{n}^{\mathrm{d}}, \mathrm{x}^{\mathrm{d}}, 1^{\mathrm{d}}\right)$, and are characterized by a negative basic unit $1^{d}=-1^{\dagger}=-1$, isodual numbers $n^{d}=$ $n 1^{d}$ and isodual product $n^{d} \times{ }^{d} m^{d}=n^{d}\left(1 / 1^{d}\right) m^{d}=n m 1^{d}$.

Following the identification of the desired numbers, Santilli passed to the systematic construction of the isodual image of all main mathematics used for the study of matter, including functional analysis, differential calculus, metric spaces, Lie algebras, symmetries, Euclidean, Minkowskian and Riemannian geometries, etc. These isodual formulations were first presented in the mathematical memoir [22] and first treated systematically in monographs [8]. The resulting mathematics is today known as Santilli isodual mathematics. It may be of some value to indicate that isoduality is a new transformation not reducible to parity and/or other conventional transformations. We should also recall the new symmetry identified by the isodual mathematics, called isoselfduality [8, 22], namely, the invariance under the isodual transformation, which is verified by the imaginary number $\mathrm{i} \equiv$ $\mathrm{i}^{\mathrm{d}}$ as well as by Dirac's equation.

Contrary to a possible perception of mathematical complexities, the isodual mathematics needed for applications can be constructed via the application of the simple anti-Hermitean map $\mathrm{Q} \rightarrow \mathrm{Q}^{\mathrm{d}}=-\mathrm{Q}^{\dagger}$, provided it is applied to the totality of quantities and to the totality of their operations 
used for the treatment of matter. Readers should be alerted that, in the absence of even one isodual map, there are inconsistencies that generally remain undetected to non-experts in the field [22].

\section{Santilli's Isodual Physics}

\subsection{Apparent Lack of Visibility of Antimatter Asteroids with Sun Light}

Santilli has achieved a representation of antimatter at all possible levels, from Newtonian mechanics to second quantization and for conditions of increasing complexity, from fully conservative conditions to the most general possible irreversible non-Hamiltonian conditions, as well as hyperstructural conditions expected in possible antimatter living structures. These studies are far from trivial and have direct implications for the very safety of our planet, since they predict that antimatter asteroids are not visible with the light of our matter Sun. In fact, the studies predict that light emitted by a matter star annihilates when hitting an antimatter body without any refraction. Alternatively, the studies predict that light emitted by an antimatter star, called by Santilli isodual light, annihilates when hitting matter, thus not reaching us on Earth due to annihilation in the upper atmosphere, as it is the case for antimatter cosmic rays. In short, Santilli has initiated an entire new field called antimatter astrophysics whose primary aim is the identification of methods for the detection of antimatter stars, by noting that their isodual light is expected to annihilate even in lenses of telescopes orbiting in space, thus requiring a basically new conception of antimatter telescopes.

It should be noted that, Einstein special and general relativity have no means for differentiating between neutral matter and antimatter as expected for asteroids and stars. As a consequence, antimatter has been assumed as being nonexistent in the universe in any appreciable amount. Santilli's discoveries indicates that antimatter has not been detected because of the above indicated occurrences, namely, the annihilation of our Sun light in an antimatter asteroid, or the annihilation of light from an antimatter star in our atmosphere or in orbiting telescopes [26].

\subsection{Newton-Santilli Isodual Equation for Antimatter}

No consistent classical theory of antimatter existed prior to Santilli's research, to our best knowledge as yet. For instance, by resuming the use of the conventional associative multiplication $\mathrm{a} \times \mathrm{b}=\mathrm{ab}$, the celebrated Newton's equation,

$$
\mathrm{m} \times \mathrm{dv} / \mathrm{dt}=\mathrm{F}(\mathrm{t}, \mathrm{r}, \mathrm{v}, \ldots . .)
$$

or the celebrated Newton's gravitation law

$$
\mathrm{F}=\mathrm{g} \times \mathrm{m}_{1} \times \mathrm{m}_{2} / \mathrm{r}^{2}
$$

solely apply for matter, and have no means whatsoever to distinguish between matter and antimatter for the very simple reason that antimatter was inconceivable at Newton's times.
Prior discovery of his isodual mathematics, Santilli developed the isodual theory of antimatter that holds at all levels of study, thus restoring full democracy between matter and antimatter. In essence, in the $20^{\text {th }}$ century antimatter was empirically treated by merely changing the sign of the charge, under the tacit assumption that antimatter exists in the same space as that for matter. Thus, both matter and antimatter were studied with respect to the same numbers, fields, spaces, etc. However, a correct classical representation of antimatter required a mathematics that is antiisomorphic to that used for matter as a necessary condition to admit a charge conjugated operator image.

Santilli represents antimatter via his anti-Hermitean isodual map that must be applied to the totality of quantities used for matter and all their operations. Hence, under isoduality, we have not only the change of the sign of the charge, but also the isodual conjugation of all remaining physical quantities (such as coordinates, momenta, energy, spin, etc.) and all their operations. This is the crucial feature that allows Santilli to achieve a consistent representation of antimatter also for neutral bodies.

We have in this way the Newton-Santilli isodual equation for antiparticles that we write in the simplified form

$$
m^{d} x^{d} d^{d} v^{d} / d^{d} t^{d}=F^{d}\left(t^{d}, r^{d}, v^{d}, \ldots . .\right)
$$

where " $\mathrm{d}$ " denotes isodual map, and the same conjugation holds for gravitation too.(see below).

Note that, after working out all isodual maps, antiparticle equation (12) merely yields minus the value of the conventional equation for particles in both the l.h.s. and the r.h.s, thus appearing to be trivial. However, a most important feature of the above equation is that it defines antiparticles in a new space, the Euclid-Santilli isodual space, which is coexistent but different than our own space. The Euclidean space and its isodual then form a two-valued hyperspace. In this section we describe how Santilli showed that, starting from the fundamental equation (12), the isodual theory of antimatter is consistent at all subsequent levels, including quantization and at that level it is equivalent to charge conjugation.

\section{Motion backward in past time}

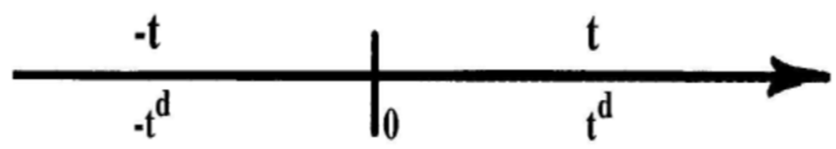

\section{Motion forward from past time}

\section{Motion forward in future time}

\section{Motion backward from future time}

Figure 1. Contrary to popular beliefs, time has four directions as depicted by Santilli in this figure to illustrate the need for isoduality. In fact, time reversal can only allow the representation of two time directions. The remaining two time directions can solely be represented via the isodual map [26].

According to Santilli the isodual antiparticles have a negative energy. This feature is dismissed by superficial 
inspections as being nonphysical, thus venturing judgments prior to the acquisition of technical knowledge. In fact, negative energies are indeed nonphysical, only when referred to our space time, that is, with respect to positive unit of time. By contrast, when referred to negative unit of time, all known objections on negative energies become inapplicable, let alone resolved.

Note also that isodual antiparticles move backward in time. This view was originally suggested by Stueckelberger in the early 1900s, and then adopted by various physicists, such as Feynman, but dismissed because of causality problems when treated with our own positive unit of time. Santilli has shown that the motion backward in time referred to a negative unit of time $\mathrm{t}^{\mathrm{d}}=-\mathrm{t}$ is as causal as motion forward in time referred to a positive unit of time $t$, and this illustrates the nontriviality of the isodual map.

Moreover, the assumption that particles and antiparticles have opposing directions of time is the only one known aspect giving hopes for the understanding of the process of annihilation of particles and their antiparticles, a mechanism utterly incomprehensible for the $20^{\text {th }}$ century physics [26].

\subsection{Isodual Representation of the Coulomb Force}

Santilli's assertion is that the isodual theory of antimatter verifies all classical experimental evidence on antimatter because it recovers the Coulomb law in a quite elementary way. Consider the case of two particles with the same negative charge and Coulomb Law

$$
\mathrm{F}=\left(-\mathrm{q}_{1}\right) \times\left(-\mathrm{q}_{2}\right) /(\mathrm{r} \times \mathrm{r})
$$

where the positive value of the r.h.s is assumed as representing repulsion, and the constant is assumed to have the value 1 for simplicity.

Under isoduality, the above expression becomes

$$
F^{d}=\left(-q_{1}\right)^{d} x^{d}\left(-q_{2}\right)^{d} /{ }^{d}\left(r^{d} x^{d} r^{d}\right)
$$

thus reversing the sign of the equation for matter, $F^{d}=-F$. However, antimatter is referred to a negative unit of the force, charge, coordinates, etc. Hence, a positive value of the Coulomb force referred to a positive unit representing repulsion is equivalent to a negative value of the Coulomb force referred to a negative unit, and the latter also represents repulsion.

For the case of the electrostatic force between one particle and an antiparticle, the Coulomb law must be projected either in the space of matter

$$
\mathrm{F}=\left(-\mathrm{q}_{1}\right) \times\left(-\mathrm{q}_{2}\right)^{\mathrm{d}} /(\mathrm{r} \times \mathrm{r})
$$

representing attraction, or in that of antimatter

$$
F=\left(-q_{1}\right)^{d} x^{d}\left(-q_{2}\right) / d\left(r^{d} x^{d} r^{d}\right)
$$

in which case, again, we have attraction, thus representing classical experimental data on antimatter [26].

\subsection{Hamilton-Santilli Isodual Mechanics}

To proceed in his reconstruction of full democracy in the treatment of matter and antimatter, Santilli had constructed the isodual image of Hamiltonian mechanics because it is essential for all subsequent steps. In this way he reached what is today called the Hamilton-Santilli isodual mechanics based on the isodual equations

$$
\begin{aligned}
& d^{d} r^{d} /{ }^{d} t^{d}=\partial^{d} H^{d}\left(r^{d}, p^{d}\right) / \partial^{d} p^{d}, \\
& d^{d} p^{d} / d^{d} t^{d}=-\partial^{d} H^{d}\left(r^{d}, p^{d}\right) / \partial r
\end{aligned}
$$

and their derivation from the isodual action $\mathrm{A}^{\mathrm{d}}$ (a feature crucial for quantization), from which the rest of the Hamilton-Santilli isodual mechanics follows [26].

\subsection{Isodual Special and General Relativities}

The special and general relativities are basically unable to provide a consistent classical treatment of antimatter. Santilli has resolved this insufficiency by providing a detailed, step by step isodual lifting of both relativities with a mathematically consistent representation of antimatter in agreement with classical experimental data. The reader should be aware that the above liftings required the prior isodual images of the Minkowskian geometry, the Poincare symmetry and the Riemannian geometry, as well as the confirmation of the results with experimental evidence [26].

\subsection{Prediction of Antigravity}

Studies on antigravity were dismissed and disqualified in the $20^{\text {th }}$ century on grounds that "antigravity is not admitted by Einstein's general relativity." According to Santilli this posture resulted in a serious obscurantism because general relativity cannot represent antimatter, thus being disqualified for any serious statement pertaining to the gravity between matter and antimatter.

With his isodual images of special and general relativity, Santilli has restored a serious scientific process in the field, by admitting quantitative studies for all possibilities, and has shown that once antimatter is properly represented, matter and antimatter must experience antigravity (defined as gravitational repulsion) because of supporting compatible arguments at all levels of study, with no known exclusion. Thereby, all known "objections" against gravitational repulsion between matter and antimatter become inapplicable under Santilli isoduality. As a trivial illustration, in Santilli's isodual theory there have the repulsive Newton-Santilli force between a particle and an isodual particle (antiparticle) both treated in our space

$$
\mathrm{F}=\mathrm{g} \times \mathrm{m}_{1} \times \mathrm{m}_{2}^{\mathrm{d}} / \mathrm{r}^{2}=-\mathrm{g} \times \mathrm{m}_{1} \times \mathrm{m}_{2} \cdot \mathrm{r}^{2}
$$

which is indeed repulsive. The same conclusion is reached at all levels of study.

Santilli further asserts that a very compelling aspect supporting antigravity between matter and antimatter is his identification of gravity and electromagnetism. In fact, the 
electromagnetic origin of exterior gravitation mandates that gravity and electromagnetism must have similar phenomenologies, thus including both attraction and repulsion [26].

\subsection{Test of Antigravity}

Santilli has proposed an experiment for the final resolution as to whether antiparticles in the gravitational field of Earth experience attraction or repulsion. The experiment consists in the measure of the gravitational force of a beam of positrons in flight on a horizontal vacuum tube $10 \mathrm{~m}$ long at the end of which there is a scintillator. Then, the displacement due to gravity is visible to the naked eye under a sufficiently low energy (in the range of the $10^{-3} \mathrm{eV}$ ). The experiment was studied by the experimentalist Mills and shown to be feasible with current technologies and resolutory $[12,26]$.

\subsection{Isodual Quantum Mechanics}

Next, Santilli constructed a step-by-step image of quantum mechanics under his isodual map based on the Heisenberg-Santilli isodual time evolution for an observable Q

$$
i^{d} x^{d} d^{d} Q^{d} / d d^{d} t^{d}=[Q, H]^{d}=H^{d} x^{d} Q^{d}-Q^{d} x^{d} H^{d}
$$

and related isodual canonical commutation rules, Schrodinger-Santilli isodual equations, etc.

He then proved that, at the operator level, isoduality is equivalent to charge conjugation. Consequently, the isodual theory of antimatter verifies all experimental data at the operator level too. Nevertheless, there are substantial differences in treatment, such as:

1) Quantum mechanics represents antiparticles in the same space of particles, while under isoduality particles and antiparticles exist in different yet coexisting spaces;

2) Quantum mechanics represents antiparticles with positive energy referred to a positive unit, while isodual antiparticles have negative energies referred to a negative unit;

3) Quantum mechanics represents antiparticles as moving forward in time with respect to our positive time unit, while isodual antiparticles move backward in time referred to a negative unit of time [26].

\subsection{Santilli's Comparative Test of the Gravity of Electrons and Positrons in a Horizontal Supercooled and Supervacuum Tube: Proposed Experiments on the Gravity of Antimatter}

The gravitational repulsion (antigravity) between matter and antimatter was suspected immediately following the discovery of antimatter, although without any possible theoretical treatment due to the absence of a theory capable of representing the gravitational field of neutral antimatter [15]. This insufficiency has been resolved by Santilli's works on antimatter. In fact, the isodual theory of antimatter predicts in a consistent and systematic way at all levels of study, from Newtonian mechanics to the Riemannian geometry, that matter and antimatter must experience gravitational repulsion
$[6,15]$.
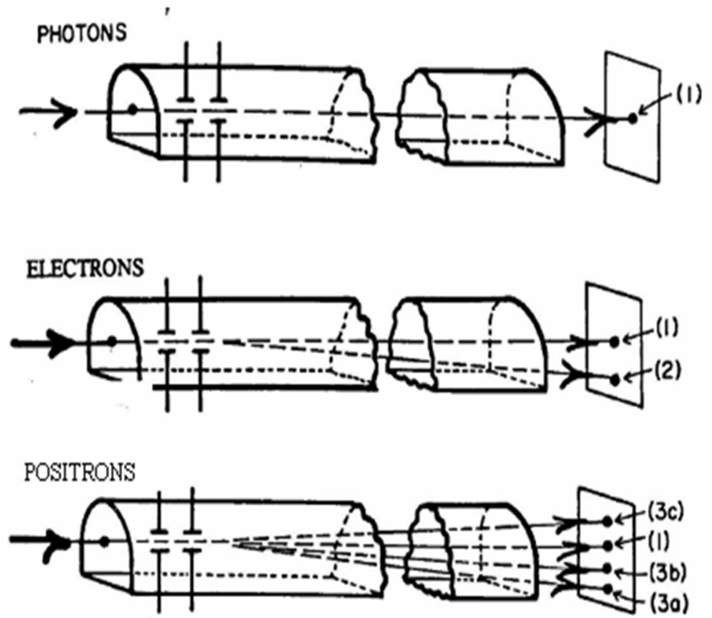

Figure 2. The original illustration used by Santilli for the 1994 proposal to test the gravity of positrons in horizontal light in a vacuum tube. The proposal has been qualified by experimentalists as being technically feasible nowadays and resolutory because the displacement due to gravity on a scintillator at the end of a $10 \mathrm{~m}$ light for positrons with milli-eV energy is visible to the naked eye. The usual criticisms based on disturbances caused by stray fields have been disqualified as political for a tube with at least $50 \mathrm{~cm}$ diameter. Virtually all major physics laboratories around the world have rejected even the consideration of the test, despite its dramatically lower cost and superior scientific relevance compared to preferred tests, on grounds that "Einstein theories do not admit antigravity, "although with documented knowledge that said theories cannot consistently represent antimatter as reviewed in the test [26].

It can conceptually said that antigravity between matter and antimatter is a necessary consequence of the very existence of a "classical" gravitational representation of "neutral" antimatter because, since the charge is null, such a representation requires the sign conjugation of all physical quantities, thus including the sign of the gravitational force and, therefore, of the curvature tensor. On quantitative grounds, we refer to monograph [15] for the gravitational representation of antigravity via the Riemannian geometry for matter and its isodual for antimatter. For this writing it may sufficient to recall the most primitive prediction of antigravity, that in Newtonian mechanics, since all subsequent levels of study are evidently compatible to such a primitive one.

In fact, the Newton-Santilli isodual equation clearly predict gravitational repulsion between matter and antimatter both in our space as well as in the isodual space, according to the respective the laws,

$$
\begin{gathered}
\mathrm{F}=\mathrm{g} \mathrm{x} \mathrm{m}_{1} \mathrm{~m}_{2}^{\mathrm{d}} / \mathrm{r}^{2}<0, \\
\mathrm{~F}^{\mathrm{d}}=\mathrm{g}^{\mathrm{d}} \mathrm{x}^{\mathrm{d}} \mathrm{m}^{\mathrm{d}}{ }_{1} \mathrm{x}^{\mathrm{d}} \mathrm{m}_{2} / \mathrm{r}^{\mathrm{d} 2 \mathrm{~d}}>0,
\end{gathered}
$$

where in our world we have a repulsion because the gravitational force is negative, $\mathrm{F}<0$, and referred to a positive unit of force, while in the isodual world we equally have a repulsion because the gravitational force is positive, $\mathrm{F}^{\mathrm{d}}>0$, but it is referred to a negative unit of force.

Safety of our planet and, consequently, this class of experiments will not be considered herein. 


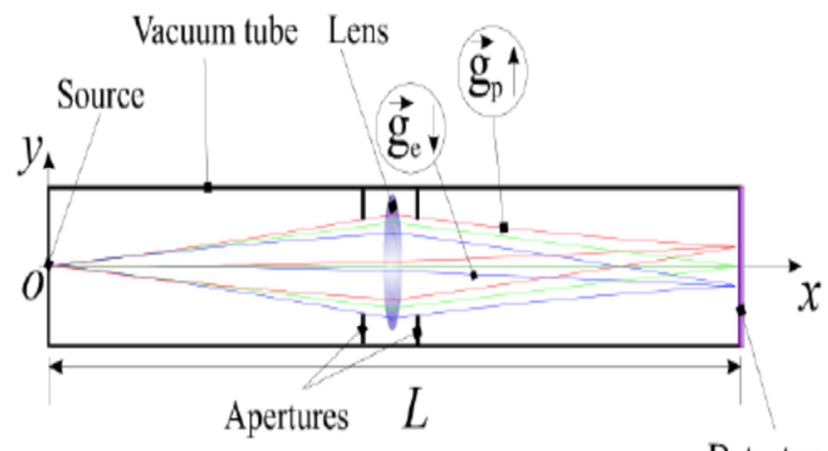

Detector

Figure 3. Principle set-up of Mills's adaptation of Santilli's comparative test of the gravity of electrons and Positrons. $\rightarrow \rightarrow$ shows the gravitational attraction on a collimated beam of electrons that, when having a very low energy of the order of meV, is of the order of $1 \mathrm{~cm}$ following a flight of $10 \mathrm{~m}$, thus being visible to the naked eye [12].

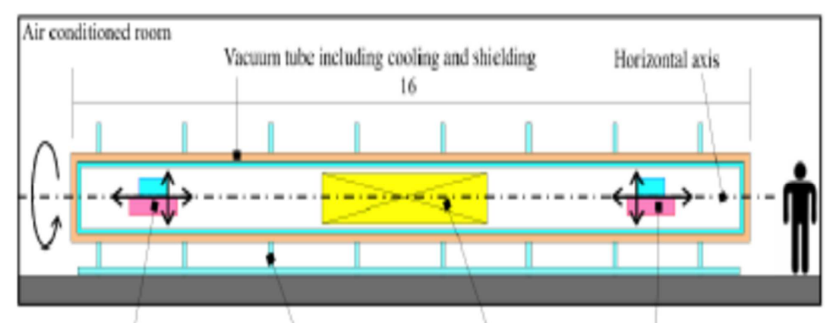

Ulira low energy pulsod source Rotation enabling support Focusing system Positioa sensitive detcctor

Figure 4. The possible alternatives for a collimated beam of positrons. Santilli's isodual theory of antimatter predicts gravitational repulsion (antigravity) at all its levels for positrons in a horizontal flight on Earth that, for very low energy of the order of meV, is of the order of $1 \mathrm{~cm}$ following a 10 $m$ flight, thus being visible to the naked eye on the scintillator at the end of the tube. For that reason, Santilli's proposed experiment has been stated to be "resolutory" by experimentalists in the field [12, 27]. The lower two renderings are from the technical realization of the test [28] by the $R . M$. Santilli Foundation on the technical realization of proposal [6] (forth view from the top) and illustration of its size compared to a person [29].

The first experimental test of the gravity of positrons was formulated by W. E. Fairbanks and E. C. Witteborn at SLAC in 1967 [30] via the use of low energy positrons in vertical upward flight in a vacuum and cooled tube. Regrettably, the experiment could not be completed due to the unavailability at that time of detectors with the extreme sensitivity needed for meaningful measurements. Numerous additional experiments have been proposed to test the gravity of positrons in vertical flights, either upwards or downwards, such as the tests of Refs. [31,32] and others. However, the gravitational force on particles is notoriously very weak, as a consequence of that the measurements with the most sophistical neutron interferometric or other techniques are expected to remain ambiguous.

Thus, the class of proposed experiments to measure the gravity of positrons in vertical flight cannot possibly be as resolutory as necessary for the

In view of the indicated limitations of testing the gravity of positrons in a vertical flight, Santilli proposed in paper [6] of 1994 the experimental verification or dismissal of the predicted gravitational repulsion between matter and antimatter via measurements of the comparative behavior of very low energy electrons and positrons moving in a $10 \mathrm{~m}$ long horizontal supercooled and super-vacuum tube (Figures 2 to 4 ).

It is evident that Santilli's gravity experiment via positrons in horizontal flight is strikingly better than preceding proposed tests [30-32] via positrons in a vertical flight. While the measurements in the latter tests are expected to remain ambiguous due to the smallness of the effect, in Santilli's experiment [6], for very low energy electrons and positrons of the order of $\mathrm{meV}$ in horizontal flight in a $10 \mathrm{~m}$ long supercooled and super-vacuum tube, the displacement due to gravity detected on a scintillator at the end of the tube is of the order of $1 \mathrm{~cm}$, thus being visible to the naked eye. The preference of Santilli's test [6] over the tests of Refs. [30-32] is confirmed by a number of experimentalists in the field. For instance, during the International Conference on Antimatter held in Sepino, Italy, in June 1996, the experimentalist A. P. Mills declared Santilli's gravity experiment as being "resolutory" [12] and, therefore, is preferable over the others not equally resolutory experiments. Similarly, during the Third International Conference on the Lie-Admissible Treatment of Irreversible Processes, held at the University of Kathmandu, Nepal, in January 2011, the experimentalist V. de Haan [27] confirmed Mills analysis and also declared Santilli's gravity experiment as being "resolutory".

Besides the above proposed experiments via the use of positrons, the only remaining proposed experiments are those based on anti-hydrogen atoms produced at CERN. Among the latter tests, we have pointed out the test proposed in Ref. [33] by the AEGIS Collaboration outlined in Figures 5 and 6, and the test proposed in Ref. [34] by the ALPHA Collaboration outlines in Figures 5 and 6 . By assuming a technical knowledge of these proposed experiments, we here limit ourselves to the following comments.

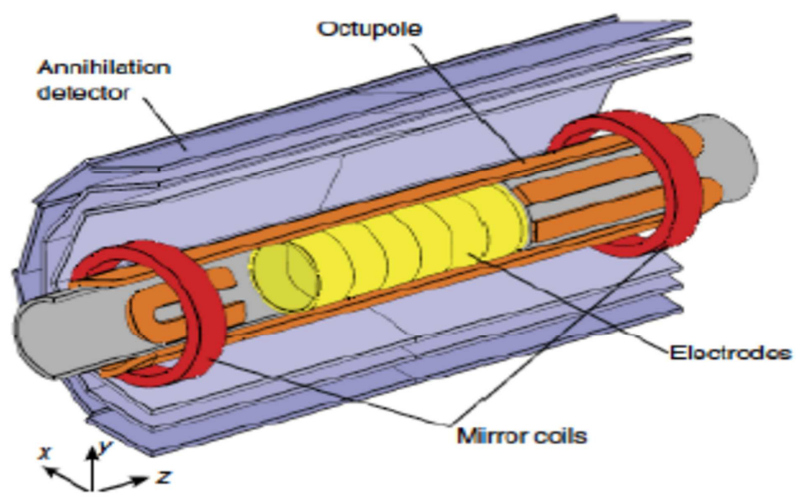

Figure 5. An illustration from Ref. [34] providing a cut-away diagram of the antihydrogen production and trapping of the ALPHA Collaboration, showing the relative positions of the cryogenically cooled Penning-Malmberg trap electrodes and other features. 


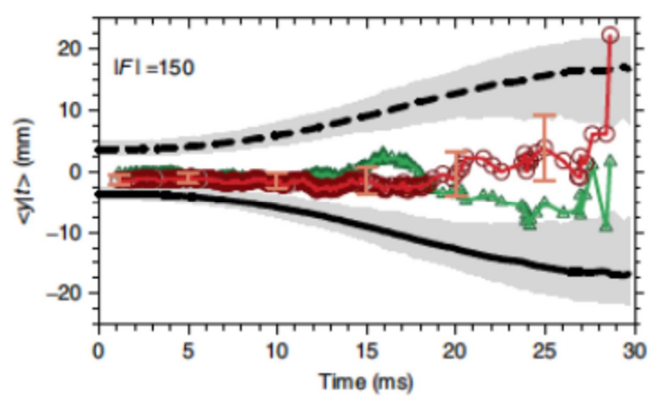

Figure 6. A second illustration from Ref. [34] on the proposed test of the gravity of antihydrogen atoms via their fall downward when released from the ALPHA antihydrogen trap of the preceding figures. The illustration depicts one of several simulated reverse cumulative average analysis. Compared to the even data to the reverse cumulative average. The green-triangle line is the reverse cumulative average of the $x$ annihilation positions of the event data, and is included as a comparison. The black solid line is the represents 900,000 simulated antihydrogen atoms. The black dashed line mirrors the black-solid line, and is equivalent to a simulation study of antigravity. The grey bands separate the $90 \%$ confidence region. Again, the complexity of the apparatus and the high sensitivity of the detectors should be compared to corresponding data of Santilli's gravity test [6,28].

To begin, the tests of Refs. [33, 34] have the same ambiguities in measurements as those of the tests with vertically moving positrons [30-32], since the former too deal with extremely small effects requiring extremely sensitive detectors under these conditions. The "experimental results" are inevitably prone to the approximations and/or manipulations that occurred in similar tests.

Besides that, the main problematic aspect of tests of Refs. $[33,34]$ is the one identified by Santilli [15] according to which, despite a popular beliefs at CERN and elsewhere, the "antiprotons" produced at CERN are not necessarily antiparticles, unless verified as such via annihilation processes, because at least in part, they can be anomalous protonic states created by the embedding of a singlet electron pairs inside ordinary protons. These states are called by Santilli the pseudoproton and denoted with the symbol $\hat{p}^{-}$.

Consequently, no gravity experiment based on "antihydrogen atoms" produced at CERN can be considered as being resolutory under such a serious ambiguity. Besides the study of antimatter, Santilli has dedicated decades of his research life also to the synthesis of neutrons inside a star according to Rutherford's historical conception that neutrons are synthesized by the "compression" of hydrogen atoms in the core of a star, nowadays represented with reaction

$$
\mathrm{p}^{+}+\mathrm{e}^{-} \rightarrow \mathrm{n}+\mathrm{v}
$$

It is well known that the energies needed to achieve the synthesis of the neutron are fully available at CERN. In particular, Santilli has shown that neutrons can also be synthesized in laboratory from a hydrogen gas traverses by a DC arc, thus taking place at energies much smaller than those available at CERN. The experimental information important for the test of the gravity of antimatter obtained by Santilli is that Rutherford's compression is also achievable for an electron pair in singlet coupling (that occurs for valence electron pairs) resulting in the creation of pseudoproton according to the reaction

$$
\mathrm{p}^{+}+\left(\mathrm{e}_{\uparrow}^{-}+\mathrm{e}_{\downarrow}^{-}\right) \rightarrow \hat{\mathrm{p}}^{-}
$$

where $\hat{\mathrm{p}}^{-}$is predicted to have a mean life essentially similar (if not longer) than that of the neutron due to the similarities of the two syntheses.

As a matter of fact, Santilli has shown that synthesis [28] is more probable than synthesis [26] for various reasons, such as: synthesis [28] does not require the emission of a neutrino for the conservation of the total angular momentum as necessary for synthesis [30], Rutherford's compression of a single electron pair inside the proton is statistically more probable than the compression of the electron due to spin zero of the electron pairs (thus requiring no special proton-pair coupling), compared to the need for a singlet proton-electron coupling for synthesis [26] and other reasons.

It should be stressed that quantum mechanics does not allow a quantitative representation of synthesis [26] because the rest energy of the neutron is bigger than the sum of the rest energies of the proton and the electron, thus requiring a "positive binding energy" which is anathema for quantum mechanics, since in this case the Schrodinger equation no longer admits physically meaningful solutions [8]. Thanks to its non-unitary invariant character, hadronic mechanics has resolved these insufficiencies by achieving, for the first time to our knowledge, a numerically exact representation of "all" characteristics of the neutrons in synthesis [26] at both non-relativistic and relativistic levels $[8,26]$.

In particular, the use of Santilli's non-unitary invariant methods that have permitted a representation of synthesis [26] when applied to synthesis [28], show that the rest energy of the pseudoproton can be close to that of the antiproton, although expecting of exact numerical values are premature at this time since the sole experimentations to date have been conducted is by Santilli.

Therefore, Santilli stresses that the distinction between the antiproton and the pseudoproton cannot be solely based on their charge and rest energy, their only resolutory distinction being that based on annihilation processes. Needless to say, the antimatter nature of the "antiprotons" claimed at CERN cannot be denied. The point is that the antimatter character has to be proved beyond doubt prior to any true scientific claim. Now, as it is well known, the production of "antiprotons" at CERN is based on hitting a target with the $26 \mathrm{GeV}$ proton beam produced by the old Proton Synchrotron (PS). It is then evident to all that, during the collision of protons with matter target, Santilli synthesis [28] is indeed possible, resulting in the synthesis of the pseudoproton. In fact, at the time of the impact, protons collide first with electrons clouds in general, including precisely the valence electron pairs of synthesis [28]. Once the pseudoproton has been synthesized, its capability to capture a positron in the anti-hydrogen trap is established by quantum mechanical laws, resulting in a neutral state $\left(\hat{\mathrm{p}}^{-} ; \mathrm{e}^{+}\right)$which is similar to, but not necessarily, the anti-hydrogen atom $\left(\hat{\mathrm{p}}^{-}, \mathrm{e}^{+}\right)$.

In short, the mathematical, theoretical and experimental 
studies illustrate Santilli's main objection against the test of the gravity of antimatter via "antihydrogen atoms" currently produced at CERN because of the lack of clear proof that they are indeed antimatter and the absence of experiments for the resolution of the ambiguities because, being necessarily beyond quantum mechanics, the said experiments are notoriously not even plausible at CERN under current control. In conclusion, both classes of tests of the gravity of antimatter, those based on vertical motion of positrons and those based on the "anti-hydrogen atoms" produced at CERN, are not resolutory on grounds of our current knowledge. Consequently, Santilli's gravity test is and remains the best measurement of the gravity of antimatter since it is the only experiment whose results would be visible to the naked eye [29].

\subsection{Experimental Detection of Antimatter Galaxies}

The isodual theory of antimatter was born out of Santilli's frustration as a physicist for not being able to ascertain whether a far away star, galaxy or quasar is made up of matter or of antimatter. Santilli has resolved this uneasiness via his isodual photon $\gamma^{\mathrm{d}}$ namely, photons emitted by antimatter that have a number of distinct, experimentally verifiable differences with respect to photons $\gamma$ emitted by matter, thus allowing, in due time, experimental studies on the nature of far away astrophysical objects.

$$
\gamma^{\mathrm{d}} \neq \gamma
$$

A most important difference between photons and their isoduals is that the latter have negative energy, as a result of which, isodual photons emitted by antimatter are predicted to be repelled in the gravitational field of matter. A possibility for the future ascertaining of the character of a far away star or quasar is, therefore, the test via neutron interferometry or other sensitive equipment, whether light from a far away galaxy is attracted or repelled by the gravitational field of Earth [26].

\subsection{The New Isoselfdual Invariance of Dirac's Equation}

Santilli has released the following statement on the Dirac equation: I never accepted the interpretation of the celebrated Dirac equation as presented in the $20^{\text {th }}$ century literature, namely, as representing an electron, because the (four-dimensional) Dirac's gamma matrices are generally believed to characterize the spin 1/2 of the electron. But Lie's theory does not allow the SU (2)-spin symmetry to admit an irreducible 4-dimensional representation for spin $1 / 2$, and equally prohibits a reducible representation close to the Dirac's gamma matrices. Consequently, Dirac equation cannot represent an electron intended as an elementary particle since elementarily requires the irreducible character of the representation. In the event Dirac's gamma matrices characterize a reducible representation of the SU (2)-spin, Dirac's equation must represent a composite system.

I discovered the isodual theory of antimatter by examining with care Dirac's equation. In this way, I noted that its gamma matrices contain a conventional two-dimensional unit $\mathrm{I}_{2 \times 2}=$ Diag. $(1,1)$, as well as a conjugate negative-definite unit $-\mathrm{I}_{2 \times 2}$. That suggested me to construct a mathematics based on a negative definite unit. The isodual map come from the connection between the conventional Pauli matrices $\sigma_{\mathrm{k}}, \mathrm{k}=1$, 2,3 , referred to $I_{2 \times 2}$ and those referred to $-I_{2 \times 2}$. In this way I reached the following interpretation of Dirac's gamma matrices as being the tensorial product of $\mathrm{I}_{2 \times 2}, \sigma_{\mathrm{k}}$ times their isoduals,

$$
\left\{\mathrm{I}_{2 \times 2}, \sigma_{\mathrm{k}}, \mathrm{k}=1,2,3\right\} X\left\{\mathrm{I}_{2 \times 2}^{\mathrm{d}}, \sigma_{\mathrm{k}}^{\mathrm{d}}, \mathrm{k}=1,2,3\right\}
$$

Therefore, I reached the conclusion that the conventional Dirac equation represents the tensorial product of an electron and its isodual, the positron. In particular, there was no need to use the "hole theory" or second quantization to represent antiparticles since the above re-interpretation allows full democracy between particles and antiparticles, thus including the treatment of antiparticles at the classical level, let alone in first quantization.

By continuing to study Dirac's equation without any preconceived notion learned from books, I discovered yet another symmetry I called isoselfduality, occurring when a quantity coincides with its isodual, as it is the case for the imaginary unit $i^{d}=i$. In fact, Dirac's gamma matrices are isoselfdual,

$$
\gamma_{\mu}{ }^{\mathrm{d}}=\gamma_{\mu}, \mu=0,1,2,3 .
$$

This new invariance can have vast implications, all the way to cosmology, because the universe itself could be isoselfdual as Dirac's equation, in the event composed of an equal amount of matter and antimatter. In conclusion, Dirac's equation is indeed one of the most important discoveries of the $20^{\text {th }}$ century with such a depth that it could eventually represent features at the particle level that actually hold for the universe as a whole [26].

\subsection{Dunning-Davies Thermodynamics for Antimatter}

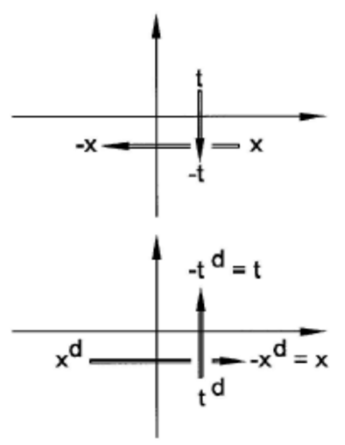

Figure 7. A schematic view of the additional peculiar property that the projection in our spacetime of the isodual space inversion appears as a time inversion and vice versa. In fact, a point in the isodual spacetime is given by $\left(x^{d}, t^{d}\right)=(-x,-t)$. The projection in our spacetime of the isodual space inversion $\left(x^{d}, t^{d}\right) \rightarrow\left(-x^{d}, t^{d}\right)$ is then given by $(x,-t)$, thus appearing as a time (rather than a space) inversion. Similarly, the projection in our spacetime of the isodual time inversion $\left(x^{d}, t^{d}\right) \rightarrow\left(x^{d},-t^{d}\right)$ appears as $(-x, t)$, that is, as a space (rather than time) inversion. Despite its simplicity, the above occurrence has rather deep implications for all discrete symmetries in particle physics [35]. 
As well known, the sole formulation of thermodynamics of the $20^{\text {th }}$ century was for matter. The first consistent formulation of thermodynamics for antimatter has been reached by J. Dunning-Davies with intriguing implications for astrophysics and cosmology yet to be explored, (see the original contribution by Dunning Davies quoted below) [26].

An important contribution to the isodual theory has been made by J. Dunning-Davies [35] who introduced in 1999 the first, and only known consistent thermodynamics for antimatter, here called Dunning-Davies antimatter thermodynamics with intriguing results and implications.

As conventionally done in the field, let us represent heat with $\mathrm{Q}$, internal energy with $\mathrm{U}$, work with $\mathrm{W}$, entropy with $\mathrm{S}$, and absolute temperature with T. Dunning-Davies isodual thermodynamics of antimatter is evidently defined via the isodual quantities

$$
\mathrm{Q}^{\mathrm{d}}=-\mathrm{Q}, \mathrm{U}^{\mathrm{d}}=-\mathrm{U}, \mathrm{W}^{\mathrm{d}}=-\mathrm{W}, \mathrm{S}^{\mathrm{d}}=-\mathrm{S}, \mathrm{T}^{\mathrm{d}}=-\mathrm{T}
$$

on isodual spaces over the isodual field of real numbers $\mathrm{R}^{\mathrm{d}}=$ $\mathrm{R}^{\mathrm{d}}\left(\mathrm{n}^{\mathrm{d}},+^{\mathrm{d}}, \times^{\mathrm{d}}\right)$ with isodual unit $\mathrm{I}^{\mathrm{d}}=-1$.

It is also seen that isodual differentials are isoselfdual (that is, invariant under isoduality). Dunning-Davies then has the following theorem:

THEOREM [36]: Thermodynamical laws are isoselfdual. Proof: For the First Law of thermodynamics we have

$$
d Q=d U-d W \equiv d^{d} Q^{d}=d^{d} U^{d}-d^{d} W^{d} .
$$

Similarly, for the Second Law of thermodynamics we have

$$
\mathrm{dQ}=\mathrm{T} \times \mathrm{dS} \equiv \mathrm{d}^{\mathrm{d}} \mathrm{Q}^{\mathrm{d}}=\mathrm{T}^{\mathrm{d}} \times{ }^{\mathrm{d}} \mathrm{S}^{\mathrm{d}}
$$

and the same occurs for the remaining laws.

Despite their simplicity, Dunning-Davies results [36] have rather deep implications. First, the identity of thermodynamical laws, by no means, implies the identity of the thermodynamics of matter and antimatter. In fact, in Dunning-Davies isodual thermodynamics the entropy must always decrease in time, since the isodual entropy is always negative and is defined in a space with evolution backward in time with respect to us. However, these features are fully equivalent to the conventional increase of the entropy tacitly referred to positive units.

Also, Dunning-Davies results indicate that antimatter galaxies and quasars cannot be distinguished from matter galaxies and quasars via the use of thermodynamics, evidently because their laws coincide, in a way much similar to the identity of the trajectories of particles and antiparticles of Lemma: The trajectories under the same magnetic field of a charged particle in Euclidean space and of the corresponding antiparticle in isodual Euclidean space coincide [8].

This result indicates that the only possibility known at this writing to determine whether far away galaxies and quasars are made up of matter or of antimatter is that via the predicted gravitational repulsion of the light emitted by antimatter called isodual light.

\subsection{Isoselfdual Spacetime Machine}

A "spacetime machine" is generally referred to a mathematical process dealing with a closed loop in the forward spacetime cone, thus requiring motions forward as well as backward in time. As such, the "machine" is not permitted by causality under conventional mathematical treatment, as well known.

Santilli discovered that isoselfdual matter, namely, matter composed by particles and their antiparticles such as the positronium, have a null intrinsic time, thus acquiring the time of their environment, namely, evolution forward in time when in a matter field, and motion backward in time when in an antimatter field.

Consequently, Santilli showed that isoselfdual systems can indeed perform a closed loop in the forward light cone without any violation of causality laws, because they can move forward when exposed to a matter and then move backward to the original starting point when exposed to antimatter [26].
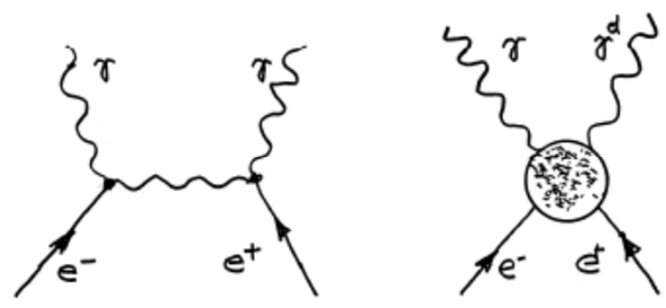

Figure 8. An illustration of the serious implications of Santilli's isodual theory of antimatter: the need for a revision of the scattering theory of the $20^{\text {th }}$ century due to its violation of the isoselfdual symmetry of Dirac's equation. The diagram in the left illustrates the isoselfduality of the initial particles (an electron and a positron) but its violation in the final particles (two identical photons). The diagram in the right illustrates one of the several needed revisions, the use for final particles of a photon and its isodual as a necessary condition to verify the new isoselfdual symmetry. Additional dramatic revisions are due to the purely action-at-a-distance, potential interactions of the conventional scattering theory (represented with a waving central line in the left diagram), compared to the non-Hamiltonian character of the scattering region caused by deep penetrations of the wave packets of particles (represented with a circle in the right diagram) [26].

\subsection{Original Literature}

Santilli's first paper on the isodual theory of antimatter is the one dating to 1994 [37] (following the 1993 paper on isodual numbers).

The first presentations of the classical isodual theory, antigravity, the isodual photon and the isoselfdual spacetime machine appeared in papers [3, 9, 38, and 39]. An independent study by an experimentalist on the feasibility and resolutory character of the proposed measurements of the gravity of positron in horizontal flight on Earth can be found in paper [12].

Comprehensive presentations of the isodual theory of antimatter are available in the monographs $[8,11]$. The first formulation of thermodynamics for antimatter was reached by J. Dunning Davies in paper [35, 40]. 


\subsection{Main Features of Santilli's Isodual Theory of Antimatter}

Santilli initiated systematic applications of isodual mathematics to the study of antimatter resulting in the new theory today called isodual theory of antimatter (or Santilli's Isodual Physics) as one of the branches of the broader hadronic mechanics [11, 26]. A main feature is that all quantities that are positive (negative) for the study of matter become negative (positive) for the study of antimatter, with the clarification that all positive and negative matter quantities are referred to positive units of measurements for matter, while all negative and positive antimatter quantities are referred to negative units. In particular, antimatter is predicted to have negative energy $\mathrm{E}^{\mathrm{d}}=-\mathrm{E}$ exactly as conceived by Dirac [41] and evolve along a negative time $\mathrm{t}^{\mathrm{d}}=-\mathrm{t}$ according to an old attempt to understand annihilation of matter and antimatter. Causality and other physical problems are resolved by the isodual mathematics, since negative quantities are measured in terms of negative units. Hence, antimatter evolving backward in time with respect to negative units of time is as causal as matter evolving forward in time with respect to positive units of time. The same holds for negative energy referred to negative units, and of other negative quantities.

The first known formulation of Newton equation for antiparticles is based on the Newton-Santilli isodual equations, and confirmed their verification of all known experimental data on the classical behavior of antiparticles [22].

A systematic presentation of the isodualities of Euclidean, Minkowskian and Riemannian geometries, Lie theory, rotational, Galilean, Lorentz and Poincare' symmetries, Galilean and special relativities, and other basic formulations is provided which in particular, presented the first known consistent representation of the gravitational field of an antimatter body via the Riemann-Santilli isodual geometry [8].

New isoselfdual cosmology at the limit of equal amounts of matter and antimatter, in which case all total quantities of the universe, such as total time, total mass, total energy, etc., are identically null to avoid a discontinuity at creation and set up the basis for continuous creation [23].

The light emitted by antimatter, also called isodual light, resulting in a prediction of main character for the detection of antimatter galaxies according to which antimatter light is physically different than matter light in an experimentally verifiable way. Since the photon has no charge, the only possible conjugation is that for all other physical quantities. As a result, antimatter light is predicted to possess negative energy while all other characteristics are opposite to those of matter light. In particular, antimatter light is predicted to be repelled by matter gravity (Fig. 9), thus permitting the conception of experiments, e.g. via neutron interferometry, to verify whether one of the two photons emitted in electron-positron annihilation experiences repulsion in our gravitational field [9, 42-47].

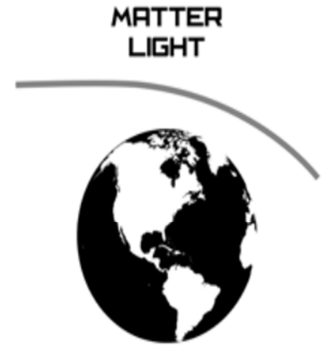

MPTTER

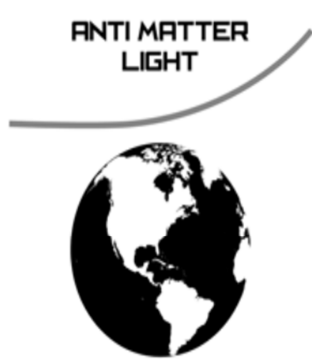

MATTER
Figure 9. A view of the repulsion of antimatter light by a matter gravitational field predicted by the isodual theory of antimatter: The repulsion of antimatter light by a matter gravitational field which is a consequence of the classical conjugation of neutral matter into antimatter.

The first known hypothesis presented that the antimatter light possesses a negative index of refraction $n^{d}=-n$ when propagating within a transparent matter medium. Again, the consistent characterization of neutral antimatter requires the conjugation of all quantities with no exclusion to avoid catastrophic inconsistencies. This implies the necessary conjugation of the index of refraction into a negative value referred to our positive units of measurements since it is observed in our matter world (Fig. 10) [42-47].

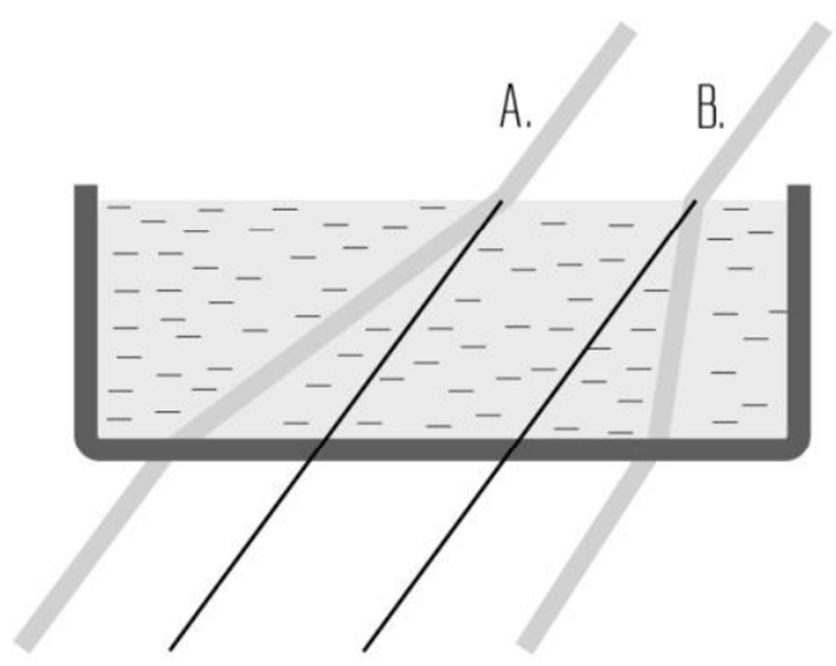

Figure 10. The prediction of negative index of refraction of antimatter light within matter water: The negative index of refraction of antimatter light which is a consequence of the repulsion of antimatter light from a matter gravitational field.

An important implication of the isodual theory of antimatter is the clarification that the conventional Dirac equation characterizes the tensorial product of one point--like particle with spin $1 / 2$ and its antiparticle without any need for second quantization [11]. Santilli could not accept the conventional $20^{\text {th }}$ century view that Dirac's equations represents only one particle with spin $1 / 2$ because there exists no irreducible or reducible representation of the $\mathrm{SU}(2)$-spin symmetry with the structure of Dirac's gamma matrices. Therefore, the author re-inspected Dirac's equation and showed that $\gamma^{\mathrm{k}}=\sigma^{\mathrm{k}} \mathrm{x} \sigma^{\mathrm{dk}}$. And $\gamma^{4}=$ Diag. $\left(\mathrm{I}_{2 \times 2},-\mathrm{I}_{2 \times 2}\right)$ thus yielding the indicated 
characterization of a spin $1 / 2$ particle and its antiparticle.

Dirac himself provided the true foundation of the isodual theory of antimatter by characterizing antiparticles with the negative unit $-\mathrm{I}_{2 \times 2}$. Dirac merely missed the mathematics for the consistent physical treatment of negative energies. Note that there is no contradiction for a representation of antiparticle at the quantum mechanical level because the isodual theory of antiparticles applies at the classical level, let alone that of first quantization.

It should be aware that a negative index of refraction implies that antimatter light propagates within a transparent matter medium at superluminal speeds. A conceptual interpretation of this prediction is that the ordinary (positive) index of refraction for matter light propagating within a transparent matter medium is due to various, ultimately attractive interactions that slow down the speed of matter light. By contrast, when antimatter light propagates within a transparent matter medium, for consistency, all features of matter have to be conjugated, resulting in new repulsive interactions between antimatter light and the matter medium that, as such, accelerate antimatter light to superluminal speeds.

\section{Application of Santilli's Isodual Theory for Detection of Antimatter Galaxies}

During his Ph. D. in physics in the mid 1960s, the Italian American scientist Ruggero Maria Santilli decided to ascertaining whether a far away galaxy was made up of matter or of antimatter and, in this way, initiated a fifty year long scientific journey. As a first step, Santilli proved that none of the $20^{\text {th }}$ century mathematics, physics and optics were applicable for a classical study of antimatter, because the annihilation of matter and antimatter into light (when in contact with each other) requires a conjugation of all physical characteristics in the transition from matter to antimatter. Such a conjugation was absent in all $20^{\text {th }}$ century sciences, since they were specifically built to treat matter. As an example, Einstein special and general relativities were conceived decades before the discovery of antimatter and, therefore, they were unable to represent matter-antimatter annihilation. Also, far away antimatter stars and galaxies have to be assumed as being neutral, thus implying the complete "inapplicability" (and not the "violation") of Einstein theories for the study of antimatter, since said theories only had the sign of the charge for conjugation.

In the early 1980s, Santilli constructed a new mathematics via a conjugation of conventional mathematics that was suitable for the "classical" description of "neutral" (or charged) antimatter bodies, technically known as anti-Hermiticity and called Santilli isoduality. Physical applications of conventional mathematics are based on positive units (such as $+1 \mathrm{sec},+1$ meter, etc.). In order to conjugate from neutral matter to neutral antimatter, Santilli constructed his new mathematics based on negative units (such as $-1 \mathrm{sec},-1$ meter, etc.). Since the charge cannot be used for conjugation of neutral bodies, Santilli achieved a consistent representation of antimatter by conjugating all physical characteristics of matter, such as mass, energy, angular momentum, etc. and by conjugating for consistency also their units.

Santilli then spent decades of studies for the construction of the isodual image of the main aspects of $20^{\text {th }}$ century mathematics, including the conjugation of number theory, functional analysis, differential calculus, symmetries, etc. The new mathematics has such a form as to admit negative left and right units at all levels [22]. The resulting new mathematics is today known as Santilli isodual mathematics [11].

Following the achievement of the appropriate new mathematics, Santilli conducted decades of studies on the construction of the corresponding physical theory, today known as Santilli isodual theory of antimatter, which includes the isodual image of all main parts of $20^{\text {th }}$ century physics, including the isodual image of special and general relativities, by achieving in particular the first known consistent classical representation of the gravitational field of neutral (or charged) antimatter bodies. Additionally, Santilli constructed the isodual image of quantum mechanics, namely, an image of quantum mechanics compatible with isodual relativities. As a central part of the above studies, Santilli proved that the isodual theory of antimatter verifies "all" known experimental data on antimatter at both the classical and quantum levels [13].

Following, decades of research for the achievement of the appropriate mathematical and physical treats, Santilli initiated experimental test of his 50 year old dream: ascertain whether a far away star or galaxy is made up of matter or of antimatter. As an invited keynote speaker at the International Conference on Antimatter held in Sepino, province of Isernia, Italy in May 1996, Santilli presented the historical discovery that light emitted by antimatter (called antimatter light) is physically different than light emitted by matter (called matter light) in an experimentally verifiable way [9]. In particular, matter light is attracted by a matter gravitational field, while antimatter light is repelled by a matter field, namely, it experiences gravitational repulsion (Figure 9).

In 2012, at International Conference on Numerical Analysis and Applied Mathematics ICNAAM in Kos, Greece, Santilli presented a second historical discovery according to which, when propagating within a matter transparent medium such as glass, antimatter light has an index of refraction opposite that of matter light (see Ref. [42] and Figure 10). This property was derived as a consequence of the gravitational repulsion of Figure 9.

This second historical discovery established that a conventional Galileo refractive telescope cannot focus images from antimatter stars because its convex lenses, such as a Steinheil achromatic convex doublet, will disperse antimatter light in all directions as shown in Figure 11. 


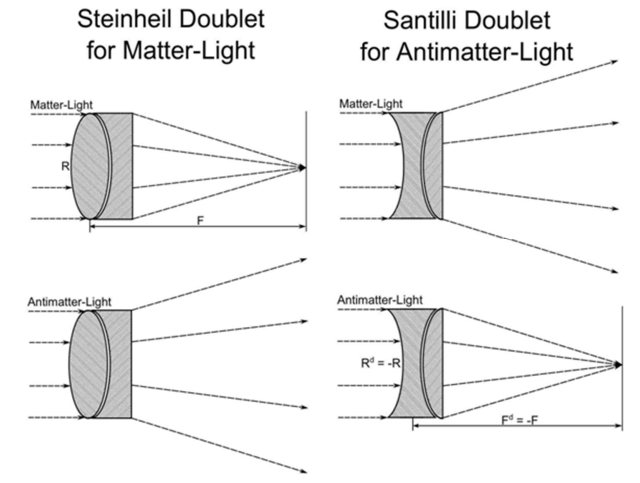

Figure 11. A view of the lenses used in the Galileo telescope to detect matter galaxies and their conjugate version used in the Santilli telescope to detect antimatter galaxies.

Consequently, Santilli conceived a conjugated doublet, called Santilli Achromatic Double Concave Doublet (international patent is pending), to focus images caused by antimatter light. Since antimatter light has an index of refraction in glass opposite that of matter light, the curvature of the lenses has to be conjugated from matter light, that is, has to be concave (see also Figures 13 and 14 of next section for details). In this way, Santilli established that none of the available telescopes can focus images of antimatter stars or galaxies because they are all based on the conventional law of refraction and related convex lenses. Consequently, images from far away antimatter stars or galaxies are dispersed in all directions by convex lenses without any focusing. Similarly, concave lenses will disperse in all directions images from mater light but they will converge images from antimatter light. Santilli also proved that we will never see antimatter images with our eyes because our iris is convex, thus dispersing antimatter light all over our retina without any focused view.

In 2012, Santilli constructed the first telescope with concave lenses, today known as Santilli Refractive Telescope or antimatter telescope (see Figure 14 of next section), and conducted systematic views of the night sky in the region of the Vega star, by achieving the first detection in scientific history of antimatter galaxies, antimatter cosmic rays and antimatter asteroids [43]. The above historical discovery has been confirmed twice by independent scientists [44] and [47].

This fifty years of mathematical, theoretical and experimental research of Santilli can provide an answer to his question of the mid 1960s, with the conclusions that: 1) All galaxies we see in the universe with the various available telescopes are solely made up of matter; 2) There exist indeed antimatter galaxies in the universe, but they are solely visible via special telescopes with concave lenses; and 3) We will never be able to focus images of antimatter with our eyes because our iris is convex.

The details of actual detections of far away antimatter galaxies and antimatter cosmic radiations are as given in the following sections.

\subsection{Santilli's Refractive Telescope with Concave Lenses or Antimatter Telescope and Experimental Method}

Santilli has been constructed a new refracting telescope with "concave" lenses; for detection of antimatter light from distant sources, because a conventional telescope with convex lenses (Galileo telescope) will disperse light with a negative index of refraction. For that Santilli secured the design and fabrication of two identical Galileo refracting telescopes; without the star diagonal viewer to avoid any unnecessary reflection of antimatter light.

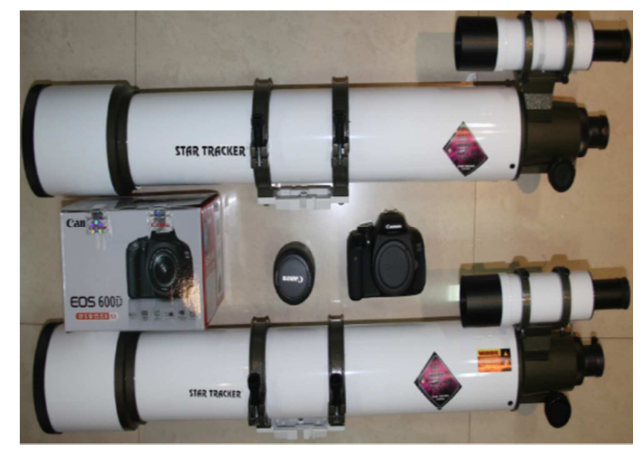

Figure 12. The two identical Galileo telescopes and the camera [43].

One of the two telescopes converted to a concave version with identical but conjugated foci. The transformation of the telescope from the Galileo form with $100 \mathrm{~mm}$ effective convex primary lenses, to the Santilli's antimatter telescope with features identical to those the Galileo one but conjugated based on Santilli's isodual mathematics as described above. Since the camera is directly attached to the telescope without the eyepiece, this conversion essentially consisted in the fabrication and assembly of concave lenses as per the data of Figure 13 and Figure 14 provides a comparative view of the Galileo and the Santilli's antimatter telescope.

He secured one single suitably selected camera (Cannon: model EOS 600D with image sensor of type CMOS, and Bayer Filter) to obtain pictures from both the Galileo and the Santilli telescopes. He also secured a tripod with mount suitable for the parallel housing of the two telescopes. He optically aligned the two telescopes on the tripod by keeping in mind the evident impossibility of doing visual alignments with the antimatter telescope and conducted a number of day views with the so mounted and aligned pair of Galileo and Santilli telescopes to verify that astronomical objects visible in the former are not visible in the latter [43].

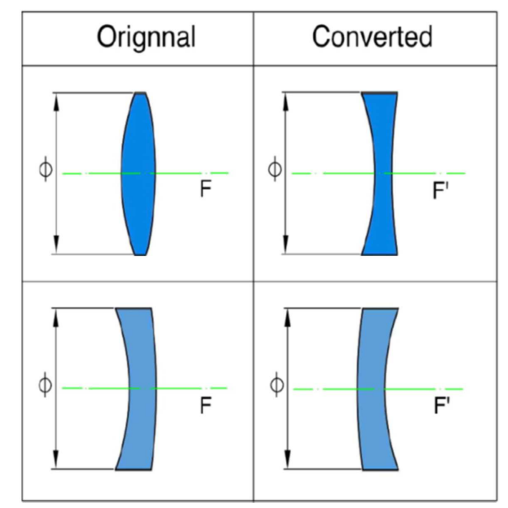

Figure 13. Main characteristics of the Galileo and antimatter primary lenses [43]. 

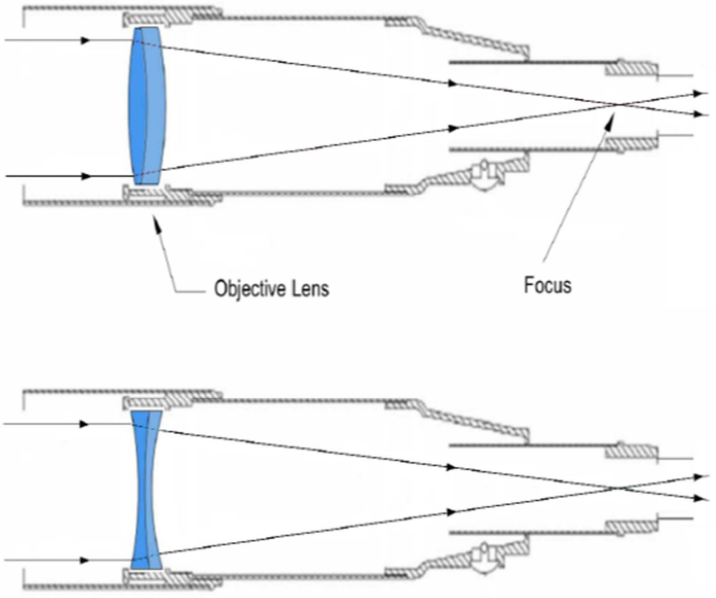

Figure 14. Schematic view of the telescopes with convex and concave lense [43].

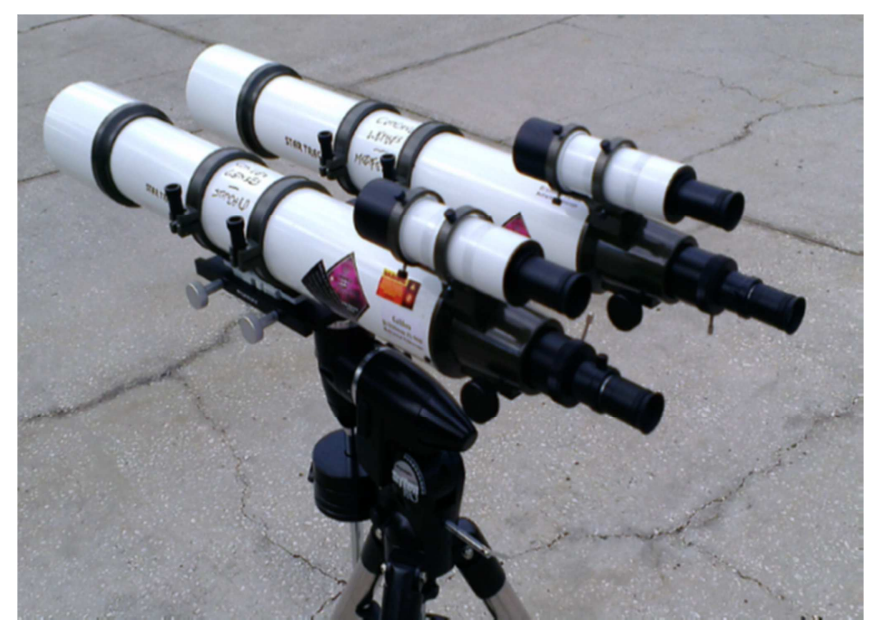

Figure 15. The parallel mount of the Galileo and antimatter telescopes with related finder scopes [43].

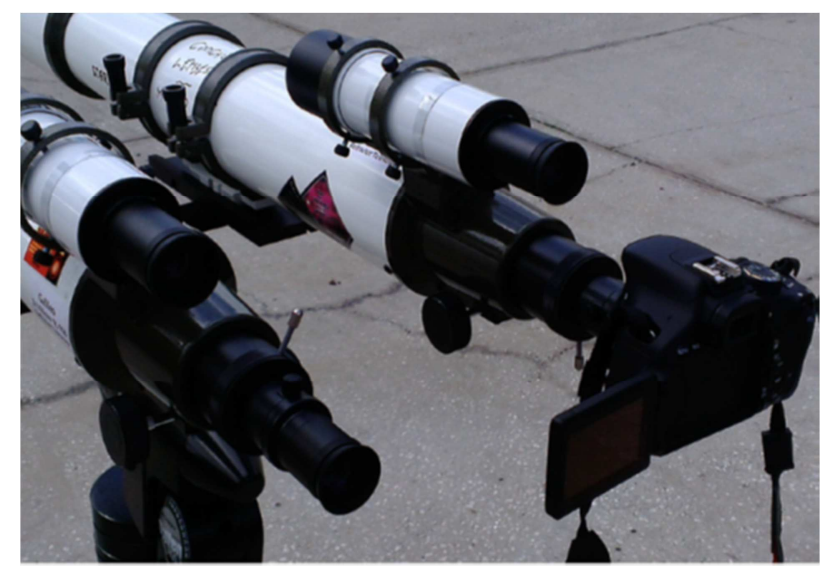

Figure 16. A close up view of the mounting of the camera directly in the telescope in place of the eyepieces [43].

A number of night views of the same region of the sky via the so mounted and aligned Galileo and Santilli telescopes was conducted and obtained a number of pictures from both telescopes via the selected camera; and finally conducted a comparative inspection of the pictures from both telescopes under a variety of enlargements and contrasts to see whether the pictures from the antimatter telescope contained focused images absent in the pictures from the Galileo telescope under the same enlargement and contrast.

Following the availability of the so mounted and aligned pair of telescopes, Santilli initiated night views by first confirming that, as expected, any celestial object visibly focused by the Galileo telescope was not focused at all with the antimatter telescope. In particular, the view of details of our Moon, which were very nicely focused by the Galileo telescope, resulted in a diffuse light when seen from the antimatter telescope without any possible identification. The same occurred for planets and nearby matter stars. Then Santilli finally initiated preliminary views of the sky at night with said pair of telescopes. He reported the tests conducted at the Gulf Anclote Park, Holiday, Florida, and GPS Coordinates: Latitude $=28.193$, Longitude $=-82.786$. The camera was set at the exposure of 15 seconds for the specific intent of having streaks of light from far away matter stars caused by Earth rotation, since streaks can be better identified with the limited capabilities of the available telescopes compared to individual dots of light in the pictures [43]. Additionally, streaks from matter stars have a clear orientation as well as length that are important for the identification of possible streaks from antimatter light. Following various tests, he selected the 10 setting of the camera at ISO 1600 because various tests with smaller and bigger ISO resulted inconclusive and ambiguous for various reasons. All pictures were analyzed (for details refer Ref. No. 43) with particular reference to the identification of the background as well as impurities in the camera sensors that are evidently present in both pictures from the Galileo and the Santilli telescope. The magnification has been obtained by Santilli via the Gimp 2.8 software [43]. In the succeeding sections pictures obtained by this pair of telescopes (Galileo telescope and Santilli telescope); in original (i.e. of Santilli) and two confirmative tests of independent researchers; are shown for the convenience of the readers. The focusing of images of antimatter galaxies, antimatter cosmic radiations etc. via a telescope with concave lenses is the first known experimental indication on the existence of antigenicity because a negative index of refraction is solely possible for the repulsion of antimatter light from matter.

\subsection{Original Pictures of Santilli's Apparent Detection of Antimatter Galaxies}

Following these preliminaries, Santilli oriented both telescopes at the indicated location and time toward the star Vega, and then specialized the orientation for the pair of matter stars Epsilon Alpha and Epsilon Beta near Vega. Some of the pictures taken by Galileo and Santilli telescopes of matter and antimatter galaxies respectively and of annihilation of antimatter cosmic rays [43] are as shown below. 


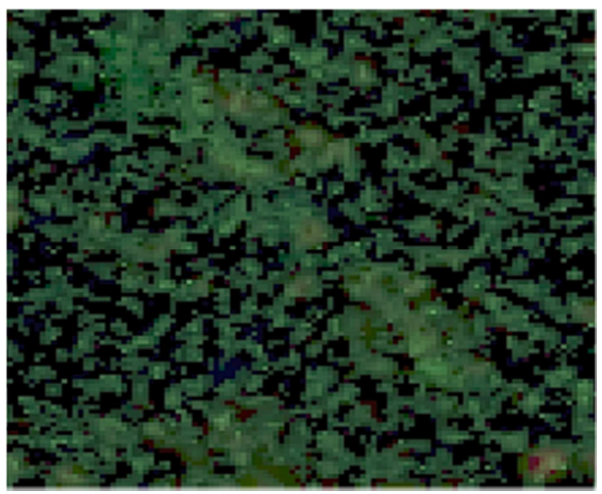

Figure 17. View of one of the streaks of matter light representing a far away matter star or galaxy identified in the Epsilon Alpha and Beta region of the night sky near Vega via the Galileo telescope [43].

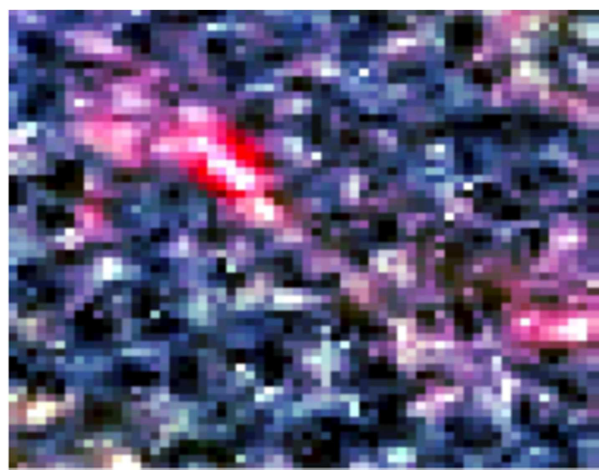

Figure 18. View of (a) First Streak of light detected in the Epsilon Alpha and Beta region with the Santilli telescope of antimatter galaxies [43].

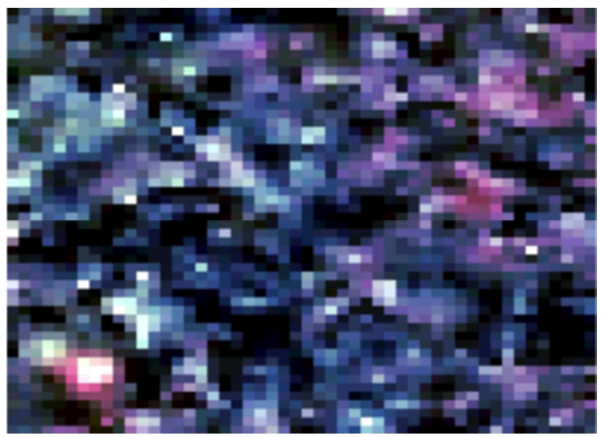

Figure 19. View of Second streak of light detected in the Epsilon Alpha and Beta region with the Santilli telescope of antimatter galaxies [43].

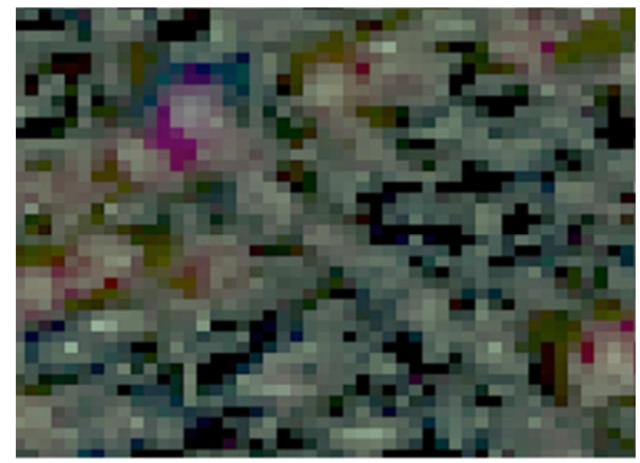

Figure 20. View of Third streak of light detected in the Epsilon Alpha and Beta region with the Santilli telescope of antimatter galaxies [43].

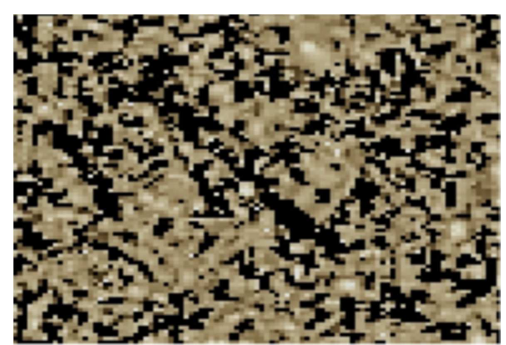

Figure 21. The first streak of darkness identified in the picture of the Epsilon Alpha and Beta region of the night sky taken with the antimatter telescope providing possible evidence of a far away antimatter star or galaxy as an alternative for the streaks of light [43].

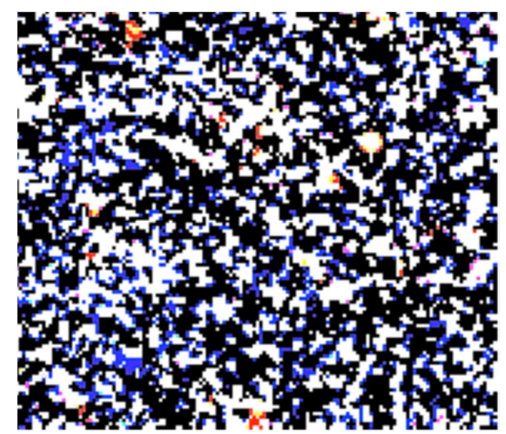

Figure 22. Another representative streak of darkness present in the antimatter telescope but absent in the Galileo telescope that may constitute an alternative to the streak of light [43].

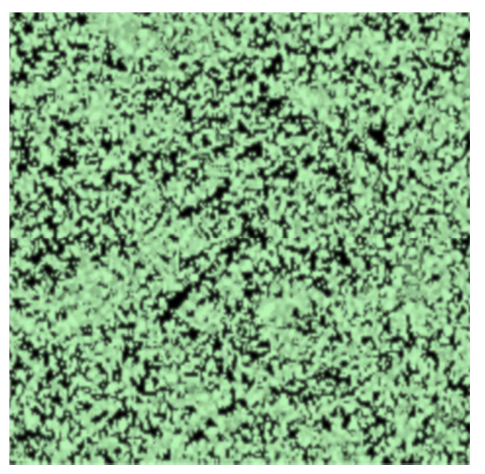

Figure 23. Seemingly connected streaks of darkness identified in a picture of the Vega region of the night sky taken with the antimatter telescope that could be due to the annihilation of a shower of small antimatter asteroids in our atmosphere, in a way much similar but the conjugate of the frequent view in the night sky of the streaks of light caused by the annihilation of a shower of small matter asteroids in our atmosphere [43].

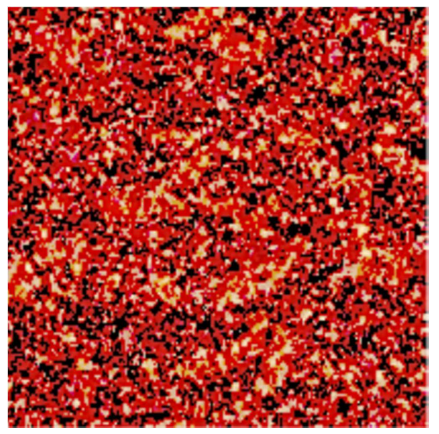

Figure 24. The first of numerous circular traces identified in a picture of Vega regions of the night sky on November 7, 2013, with the Santilli's antimatter telescope that could be due to the annihilation of an antimatter cosmic ray [43]. 


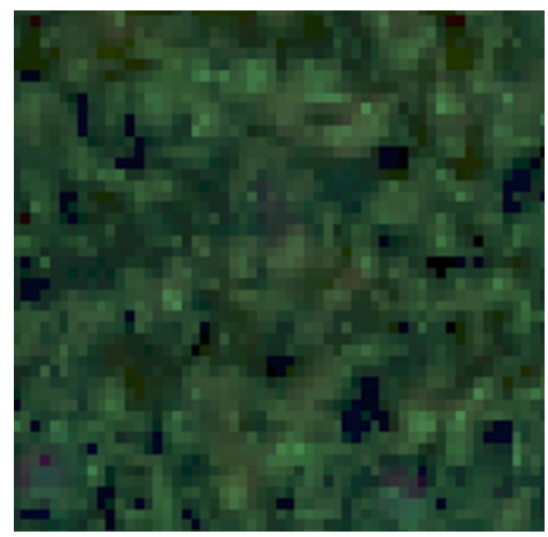

Figure 25. View of a circular trace identified in a picture of Deneb regions of the night sky with the Santilli's antimatter telescope [43].

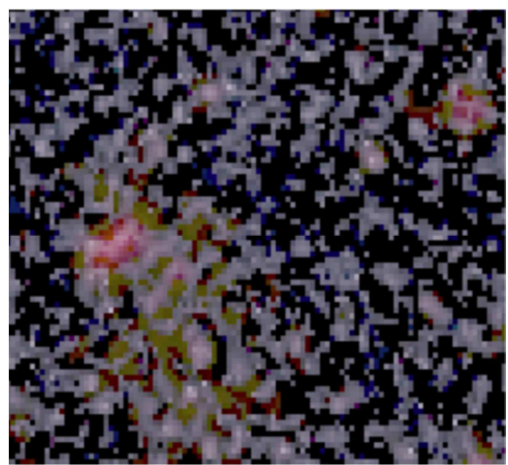

Figure 26. View of a circular trace identified in a picture of Altair regions of the night sky with the Santilli's antimatter telescope [43].

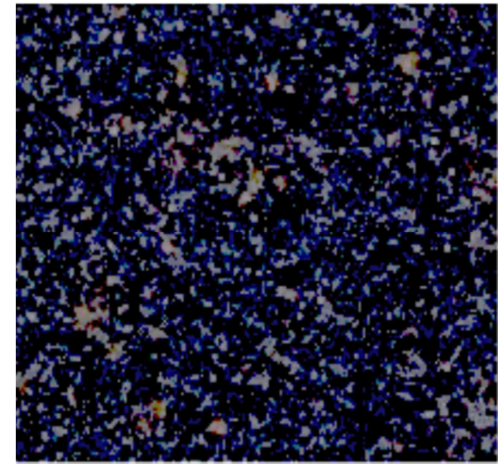

Figure 27. View of a circular trace identified in a picture of Sadr regions of the night sky With the Santilli's antimatter telescope [43].

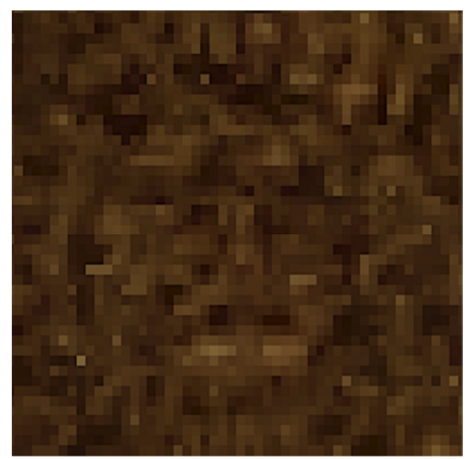

Figure 28. View of a circular trace identified in a picture of Gienah Cyngi regions of the Night sky with the Santilli's antimatter telescope [43].

\subsection{Images of Antimatter for Preliminary Confirmations of Santilli's Apparent Detection of Antimatter}

By using the same pair of Galileo and Santilli telescopes, the same camera, the same exposure of 15 seconds for ISO 1600, a team of scientist [44] went to Sebring, Florida and Enclote Gulf Park in Holiday, Florida and obtained pictures from both telescopes of the same region of the night sky studied by Santilli (that of Epsilon Alpha and Beta stars). Some of the original pictures are available from Ref. [48] in raw and tiff formats under the markings "Galileo-Epsilon-Sebring" and "Santilli-Epsilon-Sebring for readers". Figures 29 to 43 reports selected joint views from the Galileo and the Santilli telescopes showing clearly anomalous streaks that are present in the Santilli telescope but absent in the Galileo telescope, which streaks have essentially the same orientation and length of the streaks caused by matter stars, thus confirming the corresponding anomalous streaks first obtained by Santilli in a telescope with concave lenses i.e. Santilli's antimatter telescope.

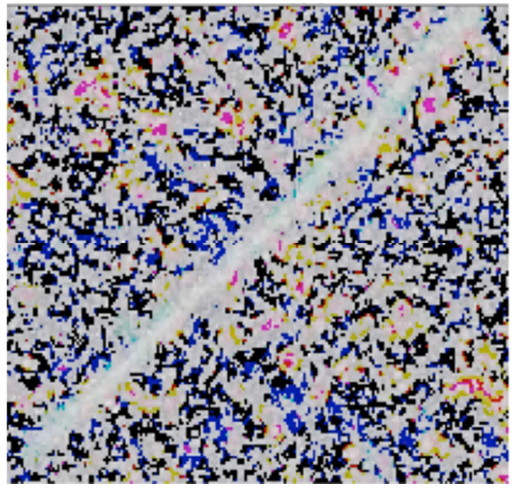

Figure 29. Picture from the Galileo Telescope of a star in the Epsilon region of the sky from Sebring, Florida [44].

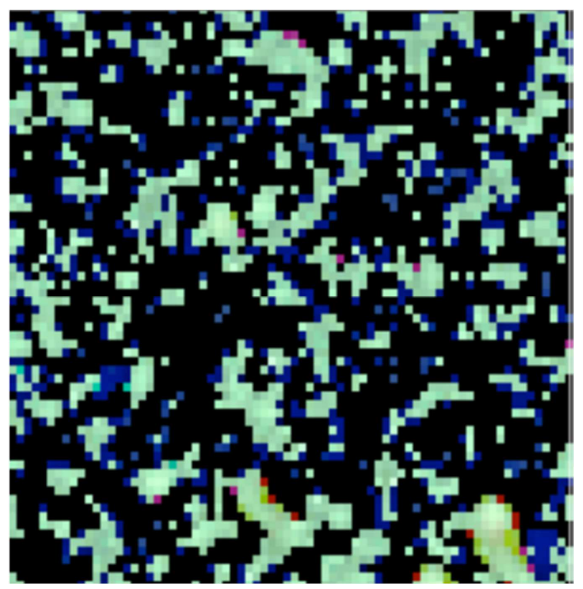

Figure 30. Picture from the Santilli telescope of a black streak in the Epsilon region of the sky from Sebring, Florida [44]. 


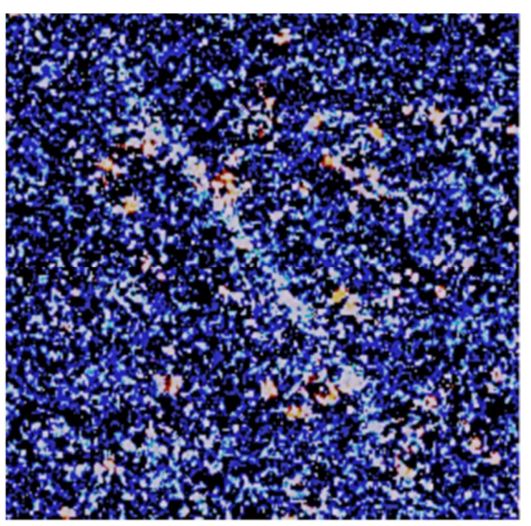

Figure 31. Picture from the Galileo telescope of a star from the Epsilon region of the sky from Sebring, Florida [44].

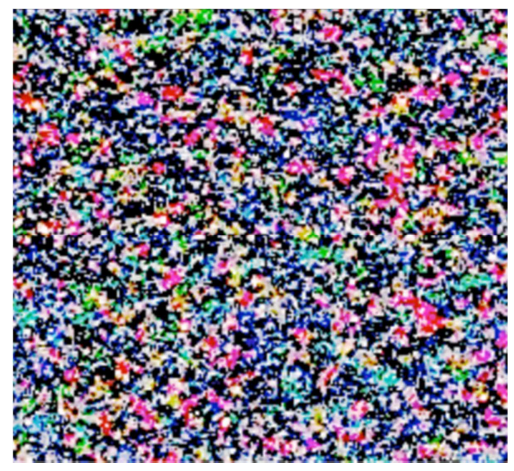

Figure 32. Picture from the Santilli telescope of a black streak in the Epsilon region of the sky from Sebring, Florida [44].

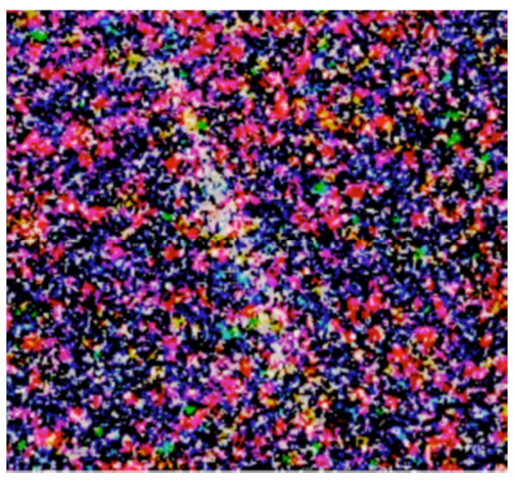

Figure 33. Picture from the Galileo Telescope of a streak of a matter star in the Vega region of the sky from Holiday, Florida [44].

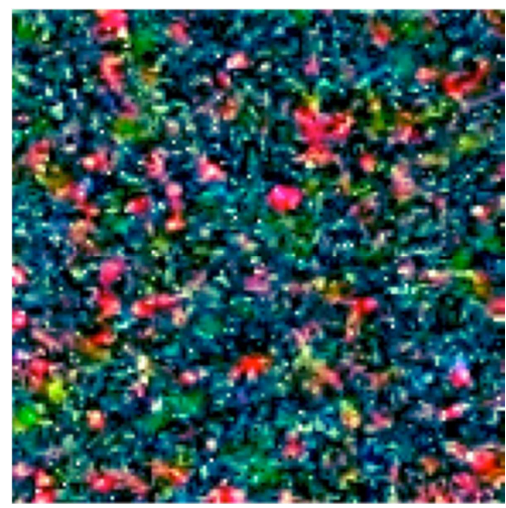

Figure 34. Picture from the Santilli telescope of a black streak in the Vega region of the sky from Holiday, Florida [44].

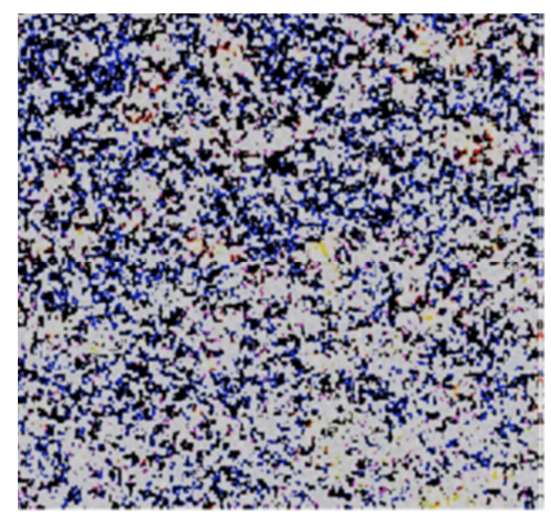

Figure 35. Picture from the Santilli telescope of another streak in the Vega region of the sky from Holiday, Florida [44].

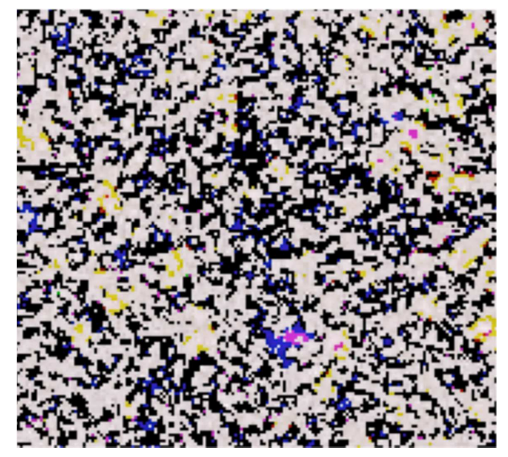

Figure 36. Picture from the Santilli telescope of an unknown event in the Epsilon sky region from Sebring, Florida [44].

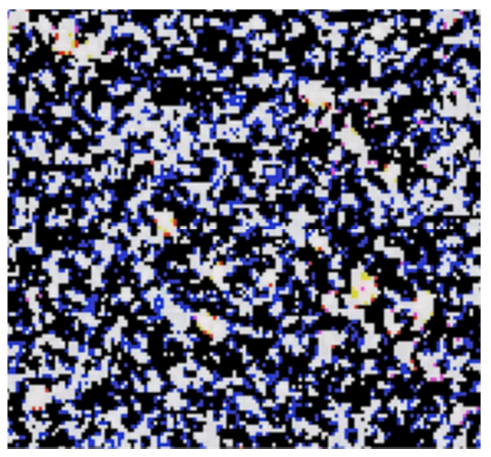

Figure 37. Picture from the Santilli telescope of a circular trace [44]

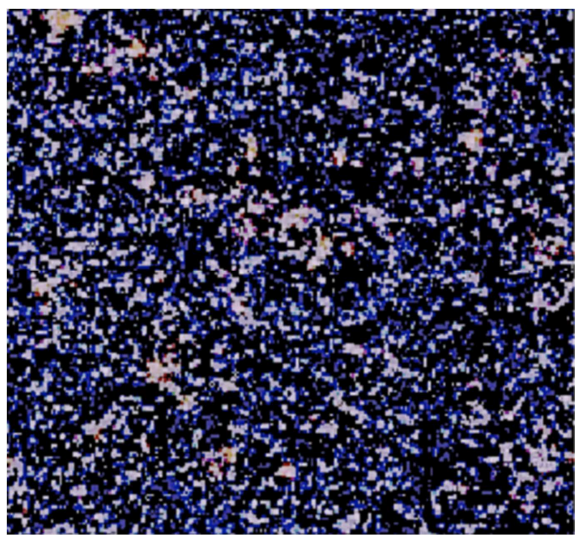

Figure 38. Picture from the Santilli telescope of another circular trace [44]. 


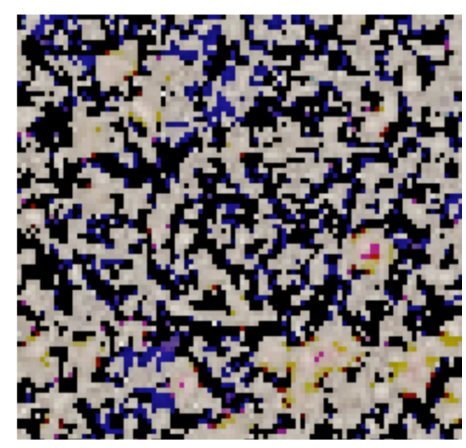

Figure 39. Picture from the Santilli telescope of yet another circular trace [44].

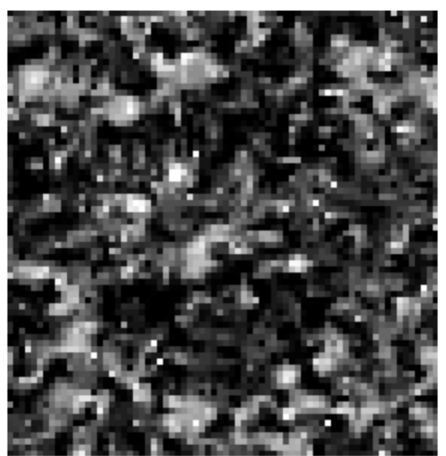

Figure 40. Picture from the Santilli telescope of yet another circular trace [44].

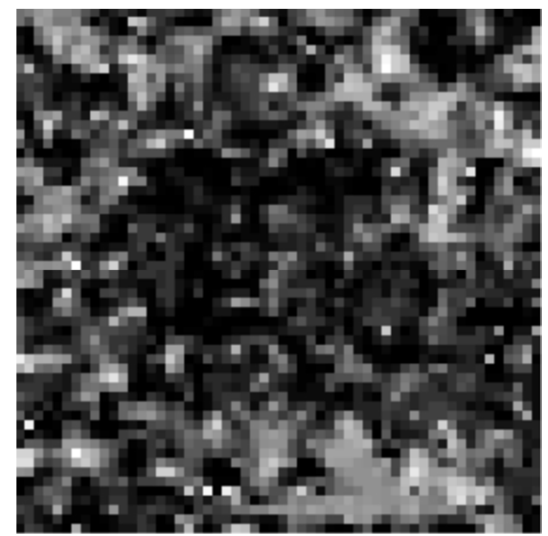

Figure 41. Picture from the Santilli telescope of yet another circular trace [44].

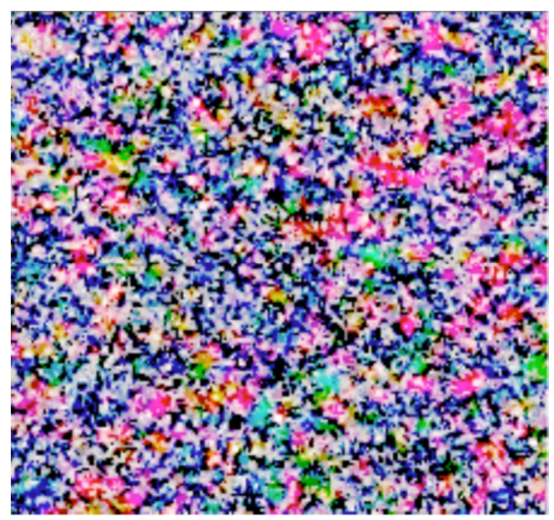

Figure 42. Picture from the Santilli telescope of yet another circular trace [44].

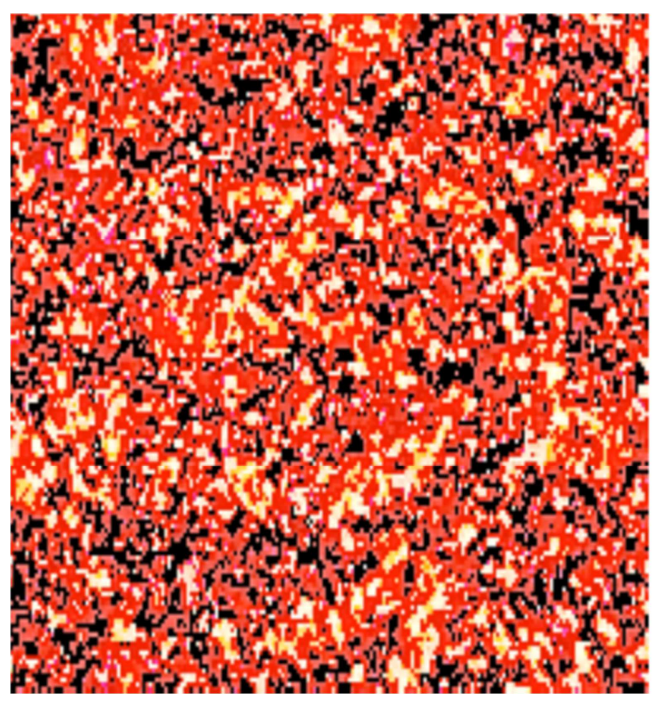

Figure 43. Picture from the Santilli telescope of yet another circular trace [44].

\subsection{Pictures of Antimatter for Confirmation of Santilli's Detection of Antimatter Galaxies}

Again some researchers [47] went to the same location of the preceding detections [43, 44], Gulf Anclote Park, Holiday, Florida, GPS: 28.193461, -82.786184 , with the same pair of parallel Galileo and Santilli telescopes, and inspected the same region of the night sky, but this time via the use of a 35 mm Canon F-IN camera with SLR film, shutter speed B, Fujifilm roll ASA 400, and exposure Compensation 1. These telescopes were oriented toward the Draco and Vega regions of the night sky under a camera exposure of both telescopes for 15 seconds and captured numerous images on $35 \mathrm{~mm}$ Fujifilm Provia 400X ISO 400 out of which, for brevity we report in the link of Ref. [49] the following images: Vega-Gal-ISO400-019.tif, Vega-Sant-ISO400-020.tif, Draco-Gal-iso200-004.tif, Draco-Sant-Iso-ISO200-005.tif. The rolls containing all original images were developed by Zebra Color Company, 1763 1st Ave. North, St. Petersburg, FL 33713 (http://zebracolor.com/index.html) and the developed images were scanned at $5760 \mathrm{dpi}$. The scanned images were enlarged and inspected via the use of paint.net software for PC. These representative images of are listed as below, in Figures 44 to 50.

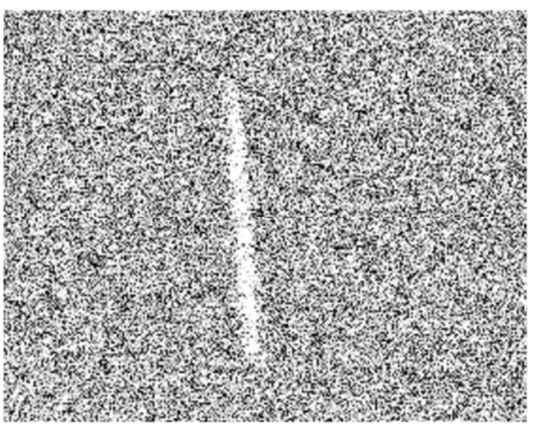

(a) 


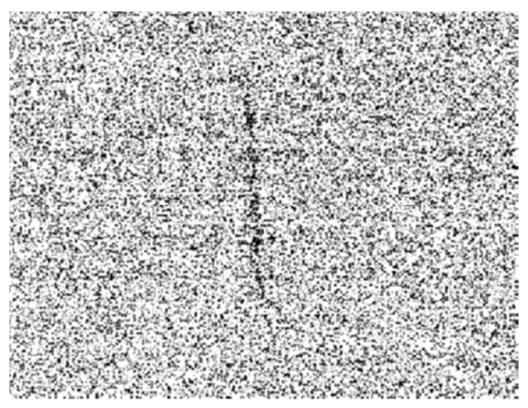

(b)

Figure 44. Images from the Vega region of the night sky showing a streak due to matter light from the Galileo telescope (a) and a streak of darkness from the Santilli telescope caused by antimatter light (b) [47].
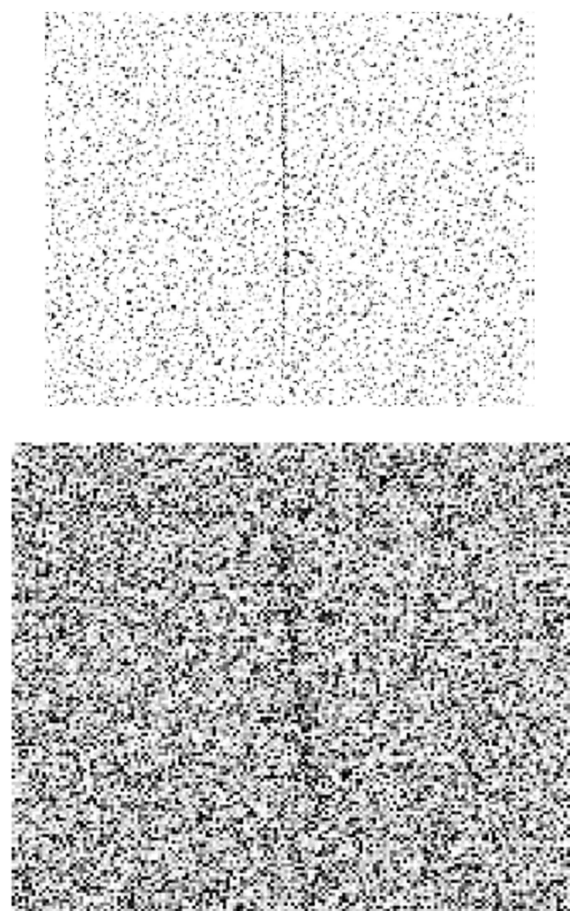

Figure 45. Images from the Vega region of the night sky from the Santilli telescope showing streaks of darkness caused by antimatter light [47].

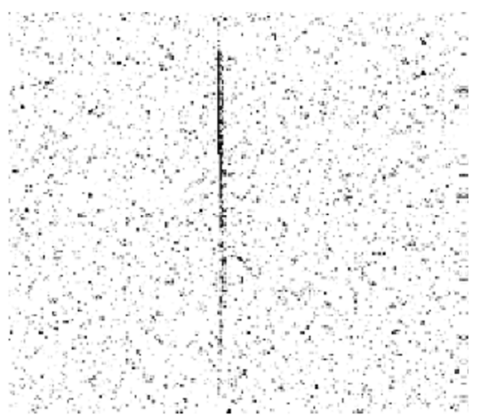

(a)

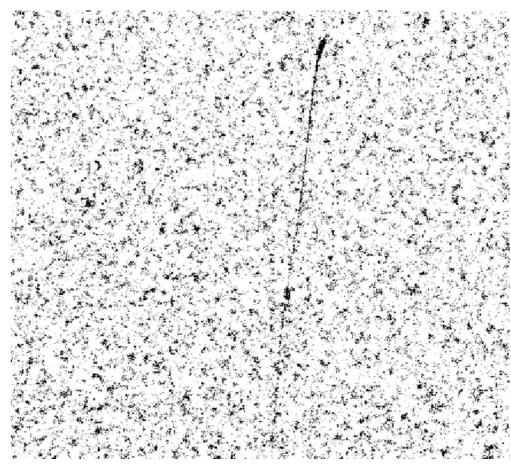

(b)

Figure 46. Images from the Vega region of the night sky showing a streak of darkness expectedly from a far away antimatter light (a), and a streak of darkness expectedly from a small antimatter asteroid annihilating in our atmosphere (b) [47].

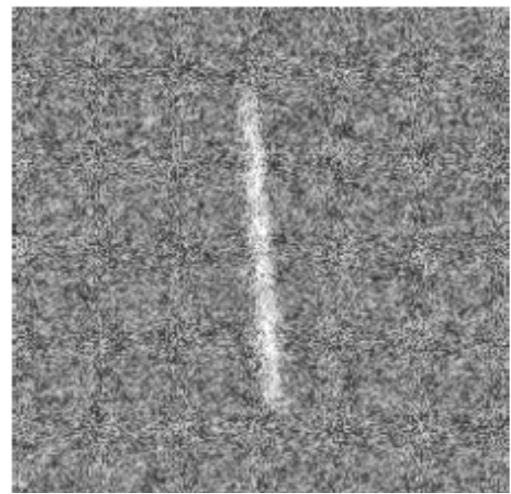

(a)

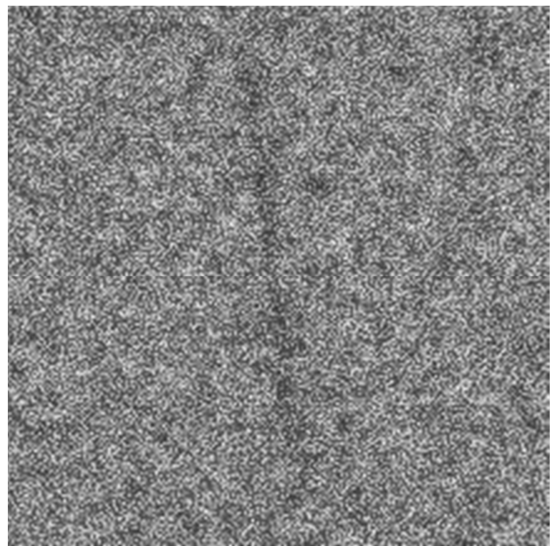

(b)

Figure 47. Images from the Draco region of the night sky showing a streak caused by matter light from the Galileo telescope (a) and a streak of darkness from the Santilli telescope caused by antimatter light (b) [47]. 

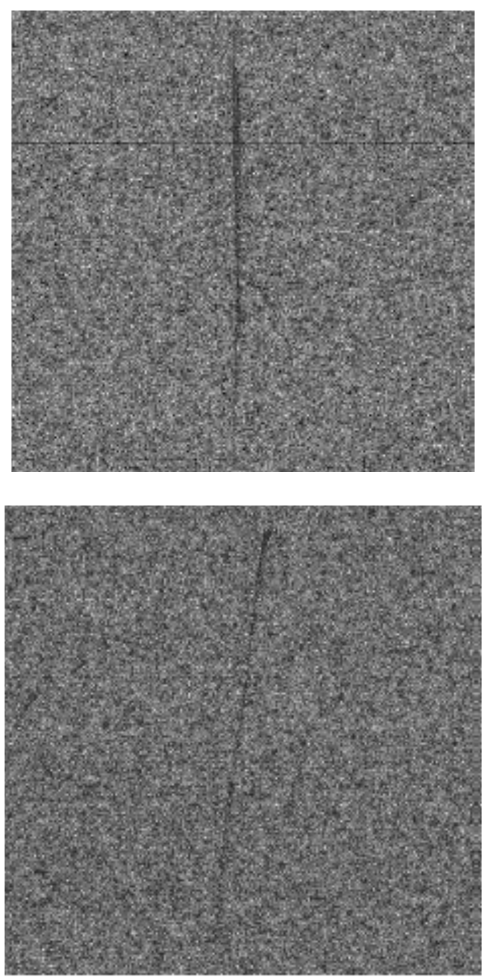

Figure 48. Images from the Draco region of the night sky via the Santilli telescope showing streaks of darkness caused by antimatter light [47].

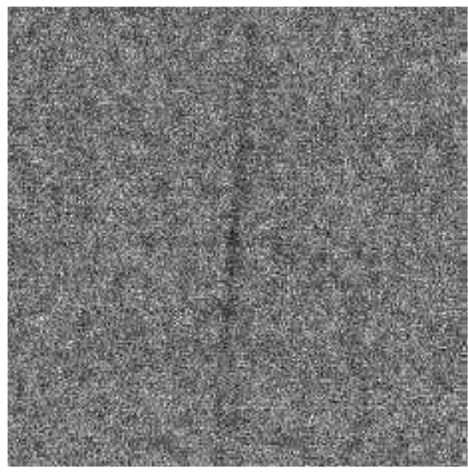

(a)

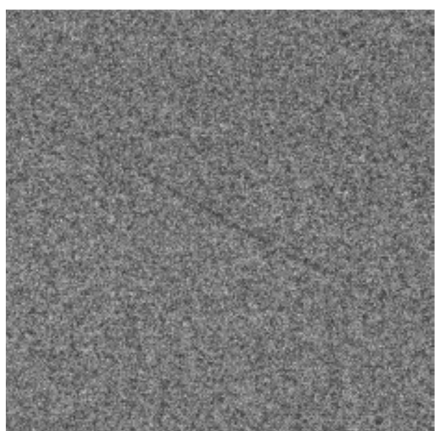

(b)

Figure 49. Images from the Draco region of the night sky showing a streak of darkness expectedly from a far away antimatter light (a), and a streak of darkness expectedly from a small antimatter asteroid annihilating in our atmosphere (b) [47].
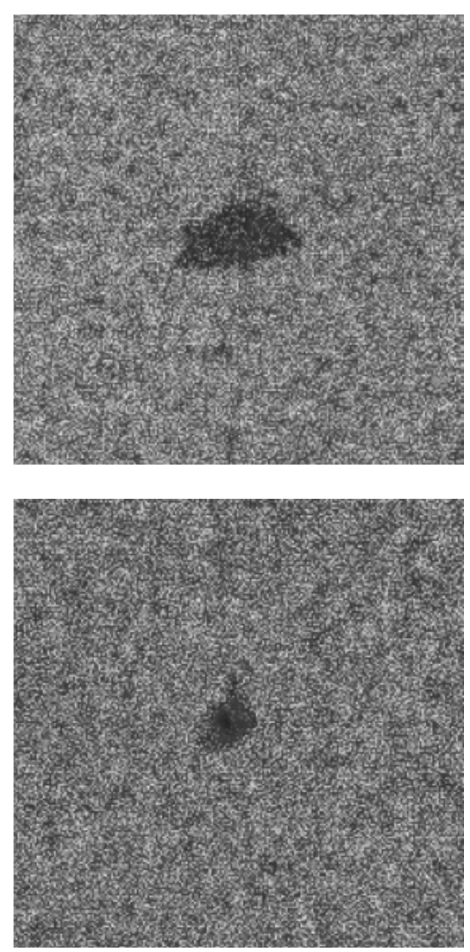

Figure 50. Images from the Draco region of the night sky via the Santilli telescope showing dots expected from the annihilation of antimatter cosmic rays in the upper regions of the atmosphere [47].

Figure 50 indicates anomalous dots (antimatter light produced by antimatter cosmic rays annihilating in the upper region of our atmosphere) present in the Santilli telescope but not in the Galileo telescope [47].

\section{Conclusion}

Based on the above analysis it is concluded that Santilli's isodual theory does indeed provide a consistent and time invariant, classical and operator description antimatter in a way compatible with available experimental data at both, the classical and quantum levels.

In particular, our analysis confirms that; 1) a consistent classical representation of neural antiparticles in a way compatible with the known quantum description can be achieved via negative-definite physical quantities such as energy, momentum, time, etc., under the consistency condition that they are measured with negative-definite units and 2) the focusing of images by a telescope with concave lenses appears to be the first experimental evidence of antigravity between matter and antimatter because the negative index of refraction for isodual light propagating within a matter medium necessary for said focusing can only be explained via repulsive interactions between the isodual light and the matter field.

\section{Acknowledgements}

The author expresses his gratitude to Prof. R. M. Santilli for encouragement and a critical reading of the paper. Thanks are also due to Prof. A. A. Bhalekar for his guidance. 


\section{References}

[1] P. A. M. Dirac, "The Principles of Quantum Mechanics", 4th ed. (Clarendon, Oxford, 1958).

[2] R. L. Forward, in Antiprotons Science and Technology, eds. B. W. Augenstein, B. E. Bonner, F. E. Mills and M. M. Nieto (World Scientic, Singapore, 1988).

[3] R. M. Santilli, A Classical Isodual Theory of Antimatter and Its Prediction of Antigravity, International Journal of Modern $\begin{array}{lllll}\text { Physics } & \text { A, } & 14 & \text { (1999) }\end{array}$ http://www.santilli-foundation.org/docs/Santilli-09.pdf.

[4] R. M. Santilli, Hadronic J. 8, (1985) 25-36.

[5] R. M. Santilli, Commun. Theor. Phys. 3 (1993) 153.

[6] R. M. Santilli, (a) Hadronic J. 17 (1994) 257; (b) Hadronic J. 17 (1994) 285

[7] R. M. Santilli, Antimatter Gravity and Antihydrogen Spectroscopy (M. Holzscheiter (editor), Baltzez Science Publ., 1997).

[8] R. M. Santilli, Elements of Hadronic Mechanics, Volumes I and II, Ukraine Academy of Sciences, Naukoa Dumka Publishers, Kiev, 1st edition (1994), 2nd edition (1995). http://www.santilli-foundation.org/docs/Santilli-300.pdf http://www.santilli-foundation.org/docs/Santilli-301.pdf

[9] R. M. Santilli, Does antimatter emit a new light? Hyperfine $\begin{array}{lllll}\text { Interactions } & \text { Vol. } & 109 & \text { (1997) 63-81. }\end{array}$ http://www.santilli-foundation.org/docs/Santilli-28.pdf.

[10] R. M. Santilli, Found. Phys. 27 (1996) 305.

[11] R. M. Santilli, Isodual Theory of Antimatter with Applications to Antigravity, Grand Unification and Cosmology, Springer (2006). http://www.santilli-foundation.org/docs/santilli-79.pdf.

[12] A. P. Mills, "Possibilities of measuring the gravitational mass of electrons and positrons in free horizontal flight", contributed paper for the Proceedings of the International Conference on Antimatter, held in Sepino, Italy, May 1996, published in the Hadronic J. Vol. 19 (1996) pp. 77-96 http://www.santilli-foundation.org/docs/Santilli-11.pdf

[13] R. M. Santilli, Isominkowskian Geometry for the Gravitational Treatment of Matter and its Isodual for Antimatter," Intern. J. Modern Phys., D 7 (1998) 351-365. http://www.santilli-foundation.org/docs/Santilli-35.pdf

[14] C. D. Anderson, Phys.Rev. 43 (1933) 491.

[15] R. M. Santilli, "Isodual Theory of Antimatter with Application to Antigravity, Grand Unification and the Spacetime Machine”, Springer, New York, (2001)

[16] P. J. Wyatt, "Possible Existence of Anti-Matter in Bulk", Nature, Vol. 181, (1958) p 1194. doi:10.1038/1811194b0.

[17] C. D. Dermer, Gamma-Ray Bursts from Comet-Antimatter Comet Collisions in the Oort Cloud."In C. Kouveliotou, M. S. Briggs, \& G. J. Fishman. 384. The Third Huntsville Symposium on Gamma-Ray Bursts, Huntsville AL, USA, October 1995, 2527, AIP Conf. Proc., Vol. 384, (1995) pp. 744-748.doi:http://dx.doi.org/10.1063/1.51650, Woodbury: American Institute of Physics.
[18] V. Rojansky, "Cosmic Rays and Comets," Phys. Rev. Vol. 58, (1940) p 1010.

[19] R. M. Santilli, "Isonumber and genonumbers of dimension 1, 2, 4,8 , their isoduals and pseudoduals, and hidden numbers of dimension 3, 5, 6," 7, Algebras, Groups and Geometries 10, (1993) pp. 273-321. http://www.santillifoundation. org/docs/Santilli-34.pdf

[20] R. M. Santilli, "Nonlocal Integral axiom-preserving isotopies and isodualities of the Minkowskian Geometry", in The Mathematical Legacy of Hanno Rund, J.V. Kadeisvili, Editor, Hadronic Press, Palm Harbor, (1993) pp. 383-430.

[21] R. M. Santilli, “Isotopies, genotopies and isodualities of Lie's Theory", Talk delivered at the International Congress of Mathematicians, Zurich, August 3-11, unpublished (1994)

[22] R. M. Santilli, "Nonlocal-Integral Isotopies of Differential Calculus, Mechanics and Geometries, in Isotopies of Contemporary Mathematical Structures" Rendiconti Circolo Matematico Palermo, Suppl. 42 (1996) pp. 7-82. http://www.santillifoundation. org/docs/Santilli-37.pdf

[23] R. M. Santilli, "A new cosmological conception of the universe based on the isominkowskian geometry and its isodual", Part I pages 539-612 and Part II pages Contributed paper in Analysis, Geometry and Groups, A Riemann Legacy Volume, Volume II, H.M. Srivastava, Editor, (1993) pp. 539- 612

[24] R. M. Santilli, "Can antimatter asteroids, stars and galaxies be detected with current means?" Proceedings of the Third International Conference on the Lie-Admissible Treatment of Irreversible Processes, C. Corda, Editor, Kathmandu University, Nepal, (2011) pp 25-36. http://www.santilli-foundation.org/docs/Antimatter-Asteroid.p $\mathrm{df}$

[25] Vougiouklis, "An Introduction to Santilli's Isodual Representation of Antimatter and the Ensuing Problem of Detecting Antimatter Asteroids", Numta Bulletin Issue 6-7, (2013). pp. 1-33 http://www.santilli-foundation.org/docs/Antimatter-2013.pdf

[26] Ivan Gandzha and Jerdsey Kadeisvily, "New Sciences For A New Era, Mathematical, Physical and Chemical Discoveries of Ruggero Maria Santilli", San-Marino draft dated June 28, 2011, Sankata Printing Press, Nepal, 2011. http://www.santillifoundation.org/docs/RMS.pdf

[27] V.de Haan, "Proposal for the realization of Santilli comparative test on the gravity of electrons and positrons via a horizontal supercooled vacuum tube", Proceedings of the Third International Conference on the Lie-Admissible Treatment of Irreversible Processes, C. Corda, Editor, Kathmandu University, (2011), $\quad$ pp. http://www.santilli-foundation.org/docs/deHaan-Arxiv.pdf

[28] R. Anderson, Santilli's comparative test of the gravity of electrons and positrons in a horizontal supercooled vacuum tube, Scientific document of the R. M. Santilli Foundation, February (2012). http://www.santilli-foundation.org/docs/anitgravity-experimen t-2012.pdf

[29] V. de Haan, "Santilli's comparative test of the gravity of electrons and positrons in a horizontal supercooled and supervacuum tube,"http://www.santilli-foundation.org/docs/Santilli-gravityexperiment.pdf 
[30] W. M. Fairbanks and E. C. Witteborn, Phys. Rev. Letters, Vol. 19 (1967) pp. 1049.

[31] G. testera, Hyperfine Interactions, Vol. 109 (1997) pp.333,

[32] R. Poggiani, Hyperfine Interactions, Vol. 109 (1997) pp. 367,

[33] M. G. Giammarchi et al. AEGIS Collaboration, "AEGIS at CERN: Measuring Antihydrogen Fall" in Proceedings of the Fifth Meeting on CPT and Lorentz Symmetry", held at Indiana University in Bloomington in June, 2010, http://arxiv.org/abs/1106.0209.

[34] A. E. Charman et al, ALPHA Collaboration, Description and first application of a new technique to measure the gravitational mass of antihydrogen," Nature Communications, April 2013. DOI: $10.1038 /$ ncomms 2787

[35] J. Dunning-Davies, "Thermodynamics of Antimatter via Santilli's isodualities", Foundations of Physics Letters, 12, (1999) pp. 593-599.

[36] [R. M. Santilli, contributed paper to Proceedings of the International Workshop on Modern Modified Theories of Gravitation and Cosmology, E. I. Guendelman, Editor, Hadronic Press, pages 113-169 (1998).

[37] R. M. Santilli, "Representation of antiparticles via isodual numbers, spaces and geometries", Comm. Theor. Phys., Vol. 3, (1994)

153-181.http://www.santilli-foundation.org/docs/Santilli-12.pd f.

[38] R. M. Santilli, “Antigravity”, Hadronic J., Vol. 17, (1994) 257-284 .http://www.santilli-foundation.org/docs/Santilli-113. pdf.

[39] R. M. Santilli, “Space-time machine”, Hadronic J., Vol. 17, (1994) 285-310. http://www.santilli-foundation.org/docs/Santilli-10.pdf.

[40] J. Dunning-Davies, "Isodual thermodynamics for antimatter", to be completed and uploaded http://www.santilli-foundation.org/docs/Dunning-Davies.pdf.

[41] P. A. M. Dirac, Proceedings of the Royal Society, 117(1928) pp. 610-624.

[42] R. M. Santilli. “The Mystery of Detecting Antimatter Asteroids,
Stars and Galaxies," American Institute of Physics Proceed. 2012, 1479, (2012) 1028-1032. http://www.santillifoundation.org/docs/antimatter-asteroids.pd $\mathrm{f}$

[43] Ruggero Maria Santilli, "Apparent detection of antimatter galaxies via a refractive telescope with concave lenses", Clifford Analysis, Clifford Algebras and their Applications (Cambridge, UK), Vol. 3, No. 1 (2014) pp 1-26http://www.santilli-foundation.org/docs/Antimattertelescope-2013-final.pdf.

[44] P. M. Bhujbal, J. V. Kadeisvili, A. Nas, S. Randall, and T. R. Shelke, "Preliminary Confirmations of Antimatter Detection via Santilli's Telescope with Concave Lenses", Clifford Analysis, Clifford Algebras and their Applications (Cambridge, UK), Vol. 3, No. 1 (2014) pp. 27-39. http://www.santilli-foundation.org/docs/Con-Ant-Tel-2013.pdf

[45] P. M. Bhujbal, "Santilli's Detection of Antimatter Galaxies: An Introduction and Experimental Confirmation”, AIP Conference Proceedings Vol. 1648 (2015) pp. 510005-1-510005-5. http://dx.doi.org/10.1063/1.4912710 http://www.santilli-foundation.org/docs/1.4912710(PM Bhujbal).pdf

[46] P. M. Bhujbal, "Santilli's Apparent Detection of Antimatter Galaxies: An Introduction and Experimental Confirmation", Hadronic Journal [Submitted for Publication] ISSN: 0162-5519 http://www.santilli-foundation.org/docs/bhujpalantim-2014.pdf

[47] Simone Beghella-Bartoli, Prashant M. Bhujbal, and Alex Nas, "Confirmation of Santilli's detection of antimatter galaxies via a telescope with concave lenses", American Journal of Modern Physics, Special Issue: New Science Light Path on Cosmological Dark Matters, 4(1-1) (2015) 34-41 http://www.santilli-foundation.org/docs/AntimatterDetection.pdf

[48] P. M. Bhujbal, J. V. Kadeisvili, A. Nas, S. Randall, and T. R. Shelke, "Pictures of the Epsilon and Vega sky" (785 MB) http://www.santilli-foundation.org/docs/Sebring- HolidayEpsilon-Vega.zip

[49] S. Beghella-Bartoli, P. M. Bhujbal, and A. Nas, "Original images of the detections", 1 Giga Bytes http://www.santilli-foundation.org/docs/original-images.zip. 